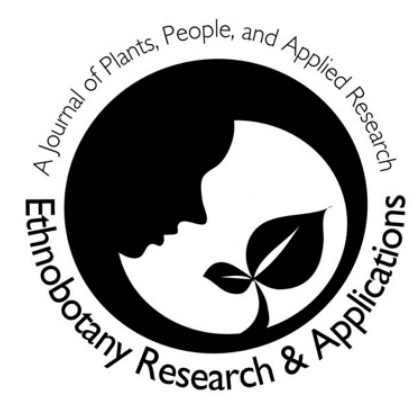

\title{
Étude ethnobotanique de Tetraclinis articulata dans la région de Béni Mellal - Khénifra
}

\author{
Ilham Zahir, Slimane Elazaoui, Mariam Chakouri, \\ Bouchera Naouer
}

\section{Research}

\begin{abstract}
Résumé
Contexte: Afin de mettre en lumière les vertus et usages traditionnels de la plante Tetraclinis articulata, une investigation ethnobotanique a été réalisée auprès de la population de la région Béni Mellal-Khénifra. L'étude a visé également de relever les connaissances de cette population à propos des effets toxiques éventuels de la plante en question.
\end{abstract}

Méthodes: Un maximum d'informations sur les différents usages et effets toxiques de $T$. articulata a été collecté via des fiches questionnaires qui ont servi d'enquêter des utilisateurs usuels de la plante et des herboristes appartenant aux différentes communes rurales et urbaines des cinq provinces de la région de Béni Mellal-Khénifra. Les questionnaires récupérés ont été dépouillés minutieusement. Les données quantitatives ont été saisies, analysées et des graphiques ont été préparés à l'aide du logiciel Excel.

Résultats: L'enquête a mis en évidence que le thuya de barbarie est utilisé principalement pour ses vertus thérapeutiques, notamment contre les affections gastro-intestinales, la fièvre et les maux de tête, soit de $26,69 \%, 25,05 \%$ et $21,07 \%$, respectivement. Cette essence végétale est également employée en cosmétique comme une lotion du corps. La majorité des remèdes sont préparés par macération à partir des feuilles et généralement administrés par voie orale sous forme de poudre sans dose précise $(59,87 \%)$. Plus de $90 \%$ des gens interrogés sont satisfaits après l'emploi des préparations à base de $T$. articulata en thérapie et en cosmétique. Par conséquent, $86,33 \%$ des enquêtés jugent que ladite plante n'est pas toxique. Cependant, $13,67 \%$ des enquêtés affirment sa toxicité en provoquant des effets secondaires tels que les affections cutanées $(25,71 \%)$ et des diarrhées $(41,42 \%)$.
Conclusion: Les plantes médicinales et aromatiques en général et de $T$. articulata en particulier doivent être utilisées avec précaution et à l'intérieur des paramètres et des mesures bien précises tout en tenant en compte de l'état physiologique de l'organisme(enfant, femme enceinte, présence d'un terrain d'allergie).

Mots clés: Tetraclinis articulata ; usages, toxicité, région Béni Mellal Khénifra, enquête.

\section{Correspondence}

Ilham Zahir*, Slimane Elazaoui, Mariam Chakouri, Bouchera Naouer

Laboratoire Polyvalent en Recherche et Développement, Département de Biologie. Faculté Polydisciplinaire Béni Mellal. Université Sultan Moulay Slimane. Mghila, Béni Mellal, 23030, Maroc

*Corresponding Author: ilham.zahir@usms.ma

\section{Ethnobotany Research \& Applications}

19:36 (2020)

\begin{abstract}
Background: In order to highlight the traditional virtues and uses of Tetraclinis articulata, an ethnobotanical investigation was carried out with the population of the Beni Mellal-Khenifra region. The study also aimed to raise the knowledge of this population about the possible toxic effects of this plant.
\end{abstract}

Methods: A maximum of information on the different uses and toxic effects of $T$. articulata was collected via questionnaire sheets which served to survey the usual users of the plant and herbalists belonging to the various rural and urban communes of the five provinces of Beni Mellal-Khénifra region. The 
questionnaires retrieved were carefully analyzed. Quantitative data was seized, analyzed and graphs were prepared using Excel software.

Results: The investigation has shown that Thuja is used mainly for its therapeutic properties, especially against gastrointestinal diseases, fever and headaches, at percentages of $26.69 \%, 25.05 \%$ and $21.07 \%$, respectively. This plant essence is also used in cosmetics as a body lotion. The majority of the remedies are prepared by maceration from leaves and generally administered orally in the form of powder without precise dose (59.87\%). More than $90 \%$ of the questioned people are satisfied after using $T$. articulata preparations in therapy and cosmetics. Consequently, $86.33 \%$ of those surveyed believe that the plant under study is not toxic. However, $13.67 \%$ of respondents affirm its toxicity by causing side effects such as skin affections (25.71\%) and diarrhea (41.42\%).

Conclusion: Medicinal and aromatic plants in general and $T$. articulata in particular must be used with caution and within very precise parameters and measures by taking into consideration the physiological state of the organism(child, pregnant woman, presence of an allergy field).

Keywords: Tetraclinis articulata; uses, toxicity, Beni Mellal Khenifra region, survey.

\section{Contexte}

Le Maroc est un pays africain situé au nord-ouest de l'Afrique sur les côtes atlantique et méditerranéenne (Zahir et al. 2019). Cette situation géographique offre au pays une richesse en niches écologiques à savoir les écosystèmes terrestres, les écosystèmes marins et côtiers ainsi que les écosystèmes des eaux continentales (Anonyme 2009).

L'ensemble de ces biotopes marocains sont caractérisés par une flore riche avec environ 7000 espèces végétales incluant 4200 taxons à flore vasculaire dont $22 \%$ (soit 879 ) sont endémiques. Parmi cette diversité botanique, 600 espèces sont connues par leurs utilisations médicinale et aromatique (Hafsé et al. 2013). Entre autres, figure Tetraclinis articulata, connue sous le nom du thuya de barbarie comme étant une espèce plus étendue et plus diversifiée au Maroc (Direction des Eaux et Forêts 1931). Cette essence forestière appartenant à la famille des Cupressacées est très employée dans la médecine traditionnelle et fournit du bois d'œuvre, du bois de service, du bois énergie, de la gomme sandaraque, du goudron et des fleurs à nectar précieux (Hajib et al. 2013, Bourkhiss et al. 2015, Zahir \& Rahmani 2020).
C'est dans cette optique que ce présent travail consiste à mettre en relief les innombrables utilisations de $T$. articulata dans plusieurs domaines thérapeutiques, cosmétiques, alimentaires et industriels grâce à une étude ethnobotanique menée auprès la population de la région Béni MellalKhénifra via la distribution des fiches questionnaires. En outre, mis à part de recenser ses maintes vertus, l'investigation a pour objectif supplémentaire de déterminer les risques toxicologiques éventuels liés à l'utilisation de la plante en question par la population sujette à l'étude. Ainsi, cette enquête ethnobotanique contribuerait à constituer une source d'information très précieuse prête à être exploitée sur le plan scientifique notamment médicale et toxicologique.

\section{Matériel et méthodes \\ Présentation de la zone d'étude}

Située au centre du Maroc, la région de Béni MellalKhénifra est née du découpage régional de 2015 à partir des trois anciennes régions:Meknès-Tafilalet, Chaouia-Ouardigha et Tadla-Azilal. Elle s'étend sur une superficie de $28.374 \mathrm{Km}^{2}$ soit $3,99 \%$ du territoire national.

Selon le Recensement Général de la Population et de l'Habitat $(\mathrm{RGPH})$ de 2014 , la région compte 2520776 habitants dont $49,14 \%$ sont urbains. La densité est de 88,8 habitants par $\mathrm{km}^{2}$, occupant ainsi la $6^{\mathrm{ème}}$ position en matière de densité. La région de Béni Mellal-Khénifra regroupe sur le plan administratif cinq provinces: Azilal, Béni Mellal, Fquih Ben Salah, Khénifra et Khouribga, 135 communes dont 16 Municipalités et 119 Communes Rurales.

Le secteur agricole est l'un des secteurs prometteurs de la région et constitue l'essentiel de l'activité économique de la région. En effet, la superficie agricole utile au niveau de la région de Béni Mellal-Khénifra est estimée à 948.426 hectares, soit $11 \%$ de la superficie agricole utile nationale. La superficie totale des terres irriguées est de l'ordre de 187.483 hectares, soit $15 \%$ de la superficie totale des terres agricoles de la région (Direction Générale des Collectivités Locales 2015).

\section{Population d'étude}

La population cible a concerné des utilisateurs de la plante $T$. articulata et des herboristes appartenant aux différentes communes rurales et urbaines des cinq provinces de la région de Béni Mellal-Khénifra. Ainsi des entrevues ethnobotaniques ont été effectuées à l'aide de fiches questionnaires qui ont servi pour enquêter cette population. Afin d'avoir une bonne représentativité de la population d'étude, un échantillonnage a été procédé d'une manière 
aléatoire et réparti en cinq stations qui correspondent chacune à une des provinces de la région de Béni Mellal-Khénifra à savoir Azilal (S1), Béni Mellal (S2), Fquih Ben Salah (S3), Khénifra (S4) et Khouribga (S5) (Tab. 1; Fig. 1).

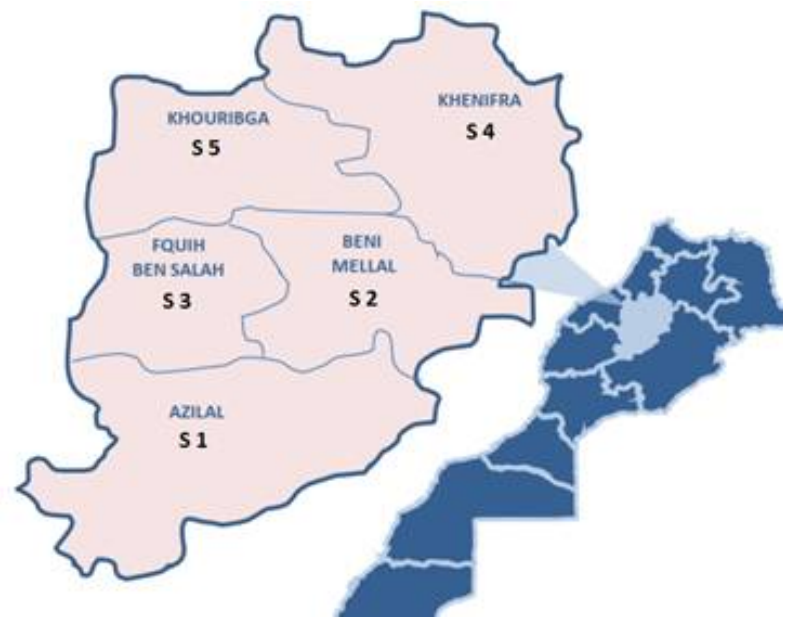

Figure 1: Présentation des cinq stations sélectionnées pour l'étude (https://asmex.org/wpcontent/uploads/2018/12/Monographie-region-deBeni-Mellal.pdf)

Figure 1: Presentation of the five stations selected for the study (https://asmex.org/wpcontent/uploads/2018/12/Monographie-region-deBeni-Mellal.pdf)

\section{Critères d'exclusion}

Les personnes n'appartenant pas à la région de Béni Mellal-Khénifra ont été exclues de l'étude.

\section{Déroulement de l'étude}

La distribution des questionnaires a été effectuée par trois enquêteurs. Les questionnés ont été invités à répondre d'une manière volontaire et anonyme. L'investigation a débuté le 27 Avril et a pris fin le 31 Mai 2019.

\section{Données recueillies}

Le contenu des questionnaires s'articule autour des rubriques suivantes renfermant une diversité de questions relatives au profilage des enquêtés, aux différents domaines d'utilisation de la plante $T$. articulata, à sa partie utilisée, à son mode de préparation et à la fréquence d'utilisation. Il est jugé judicieux également d'évaluer les connaissances des enquêtés à propos des effets désirables éventuels de la dite plante.

\section{Analyse statistique}

Les questionnaires récupérés ont été dépouillés minutieusement. Les données quantitatives ont été saisies, analysées et des graphiques ont été préparés à l'aide du logiciel Excel (Microsoft office 2007).

\section{Aspect légal}

Les enquêtés sont informés que le remplissage du questionnaire se fait à l'anonymat et que l'exploitation des données est réalisée dans un cadre strict de la recherche universitaire.

\section{Résultats et discussion}

Les résultats du dépouillement et de l'analyse des questionnaires sont présentés et discutés ci-après.

\section{Population d'étude de l'enquête}

L'enquête a été menée auprès de la population habitant les différentes communes rurales et urbaines des cinq provinces de la région Béni MellalKhénifra (Annexe 1). Ainsi, le nombre globale des enquêtés interrogés est égale à trois cent dix (310) personnes.

\section{Profilage des enquêtés \\ Age}

L'utilisation de la plante étudiée est répandue chez l'ensemble des personnes questionnées, avec une prédominance chez les personnes entre 15 et 30 ans, soit $52,24 \%$. Par ailleurs, un taux de $47,11 \%$ est noté chez les personnes âgées de 30 à 100 ans. L'utilisation est moins importante $(0,64 \%)$ chez les enfants qui ne dépassent pas l'âge de 15 ans(Fig. 2).

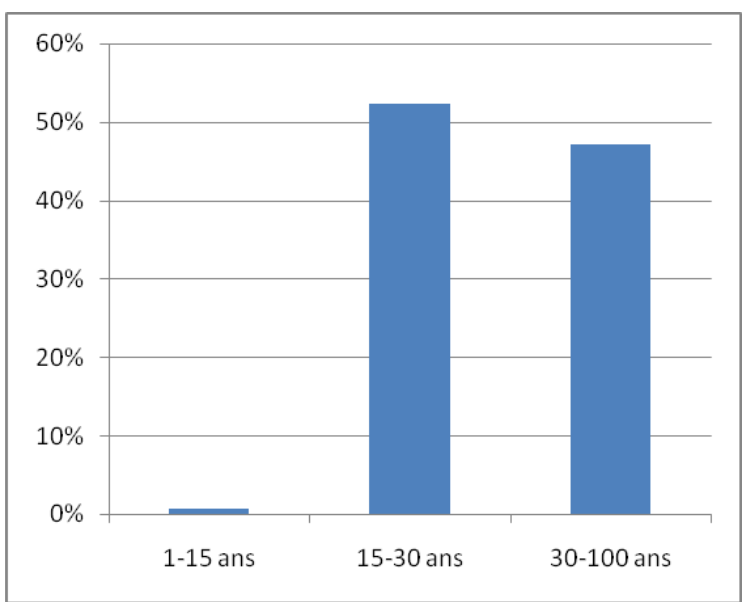

Figure 2: Répartition des enquêtés selon leur âge Figure 2: Distribution of respondents by age

\section{Sexe}

D'après la Fig. 3, on constate une dominance d'utilisation du thuya de barbarie par les femmes avec un pourcentage de $57 \%$, contre $43 \%$ chez les hommes. Ce résultat est en accord avec plusieurs études précédentes qui ont montré que les femmes représentaient la catégorie utilisant le plus les plantes médicinales (Azzi et al. 2012, Fakchich \& Elachouri 2014, Rhattas et al. 2016, Ben Akka et al. 2017, Katiri et al. 2017, Kadri et al. 2018). En fait, les femmes sont plus détentrices du savoir phytothérapique traditionnel en apprenant ce savoir 
principalement de leurs mères et grands-mères à travers des observations de routine (El Yahyaoui et al. 2015, Katiri et al. 2017). En revanche, d'autres recherches indiquent que ce sont plutôt les hommes notamment les herboristes qui sont intéressés aux vertus des végétaux (Bachiri et al. 2015, Salhi et al. 2019).

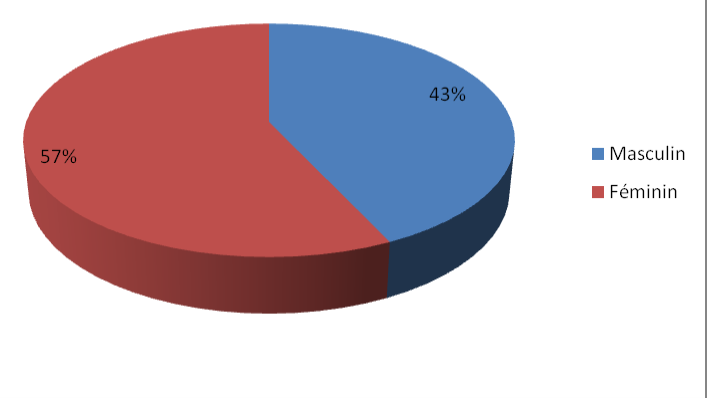

Figure 3: Répartition des enquêtés selon leur sexe Figure 3: Distribution of respondents by gender

\section{Niveau d'instruction}

Dans la zone d'étude, $18,09 \%$ des utilisateurs de la plante étudiée sont des analphabètes, alors que les $81,91 \%$ correspondent à différents niveaux intellectuels ainsi $10,19 \%, 13,81 \%$ et $51,64 \%$ des enquêtés ont bénéficié d'enseignements primaire, secondaire et universitaire, respectivement. Par ailleurs, $6,25 \%$ des personnes ont eu une éducation non-formelle (Fig. 4). Ainsi, on constate que l'enseignement universitaire arrive en tête du classement ce qui représente un résultat inhabituel en comparaison avec les aboutissements d'autres recherches ethnobotaniques découvrant que la plupart des enquêtés sont des analphabètes (Ghourri et al. 2012, Fakchich \& Elachouri 2014, Bachiri et al. 2015, El Yahyaoui et al. 2015, Rhattas et al. 2016, Ben Akka et al. 2017, Katiri et al. 2017, Chraibi et al. 2018, Kadri et al. 2018, Salhi et al. 2019). Consécutivement, ceci montre l'intérêt porté par la catégorie intellectuelle de la population d'étude de la région de Béni Mellal - Khénifra aux plantes médicinales.

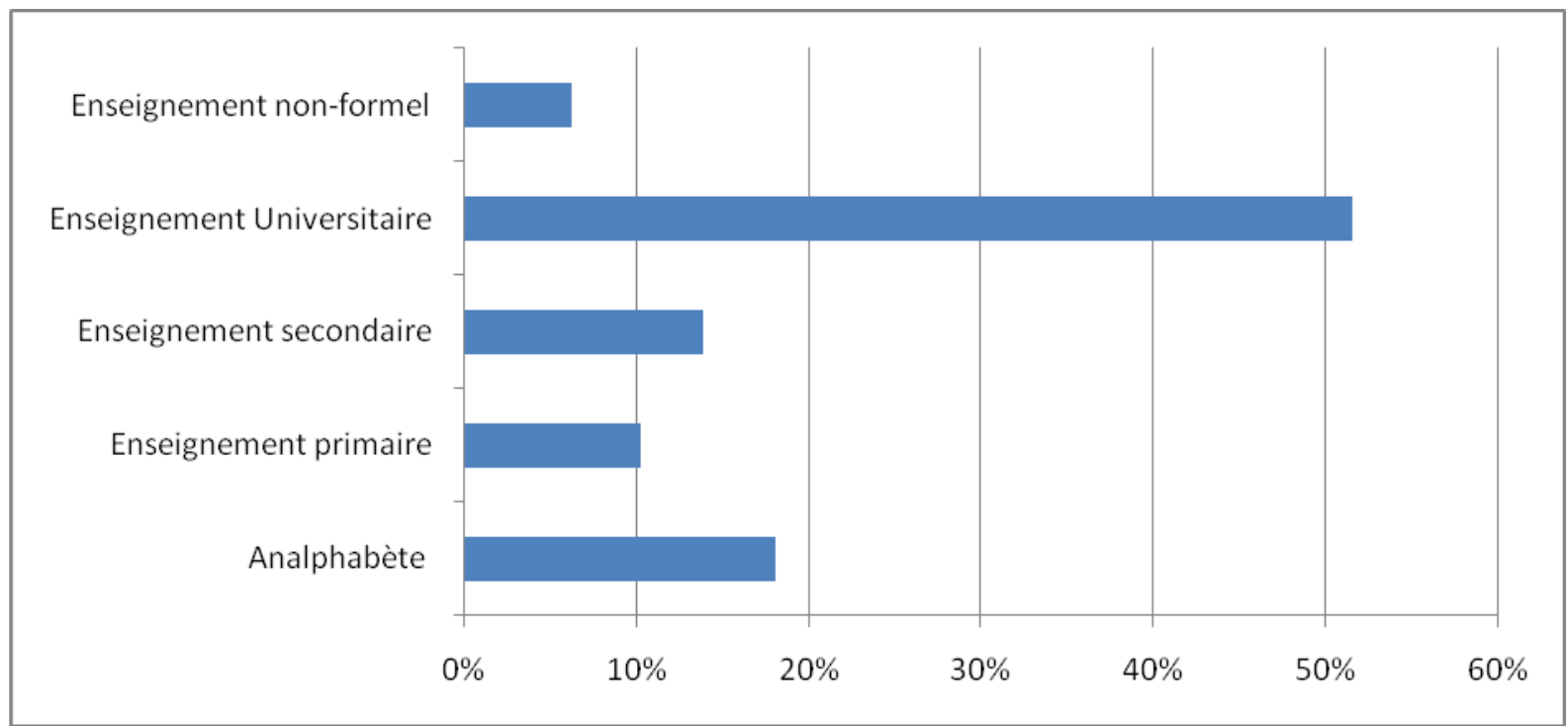

Figure 4: Répartition des enquêtés selon leur niveau d'instruction

Figure 4: Distribution of respondents according to their level of education

\section{Lieu de résidence}

La plupart des enquêtés proviennent des villages, soit $45,08 \%$, suivie par des personnes vivant dans les villes, soit $38,98 \%$. Tandis que $15,94 \%$ des enquêtés sont issus des montagnes de la région de Béni Mellal-Khénifra (Fig. 5). Ces données indiquent que les participants ruraux sont les principaux consommateurs des plantes médicinales $(61,02 \%)$ comme il a été rapporté par l'investigation dirigée par Fakchich \& Elachouri(2014). Ceci a été expliqué dans l'étude précitée par le fait que la population rurale garde un bon contact avec la nature. Une autre raison éventuellement appuyant ce résultat est que la plupart des habitants des zones rurales ont un faible revenu ne leur permettant pas de consulter un médecin et/ou d'acheter des médicaments ce qui les laisse devant la phytothérapie comme un recours moins cher pour être traités.

\section{Usage de $T$. articulata}

Connaissances à propos la plante $T$. articulata $96,44 \%$ des enquêtés ont déjà entendu à propos $T$. articulata, à l'encontre $3,56 \%$ seulement ne connaissent pas cette essence végétale (Fig. 6).

\section{Utilisation de la plante $T$. articulata}

$T$. articulata est utilisée par la majorité des enquêtés de la région de Béni Mellal - Khénifra avec un pourcentage de $85 \%$, contre $15 \%$ seulement pour les non-utilisateurs (Fig .7). 


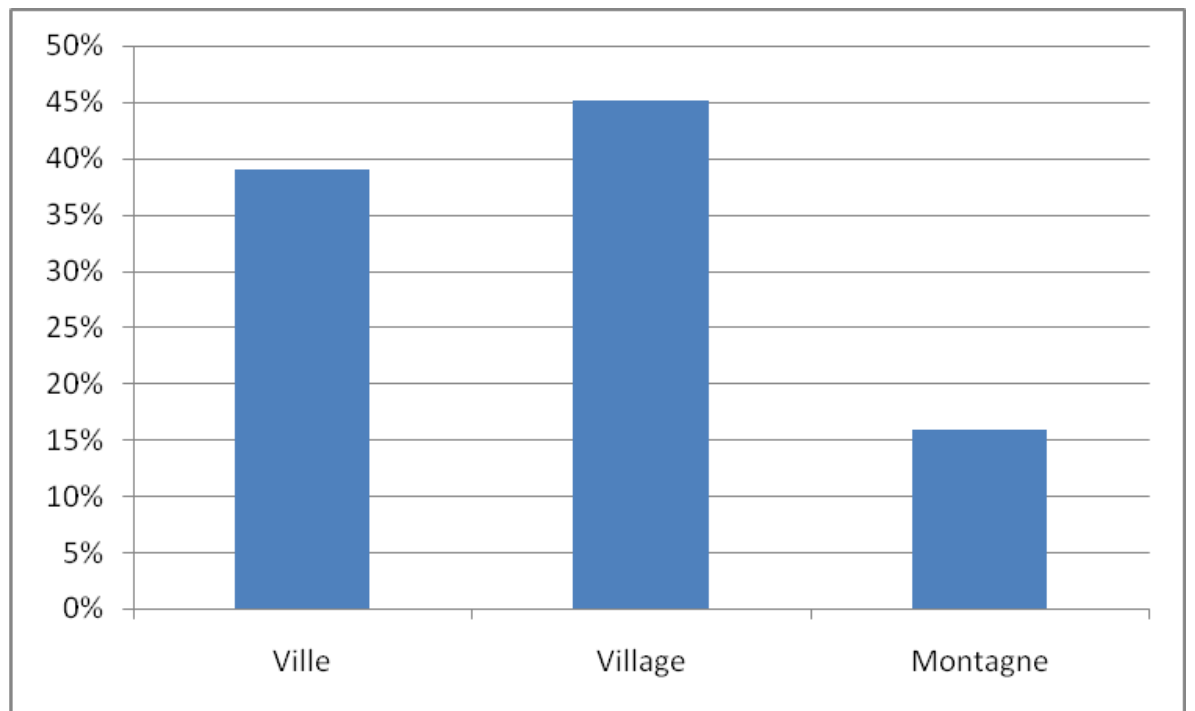

Figure 5: Répartition des enquêtés selon leur lieu de résidence Figure 5: Distribution of respondents by place of residence

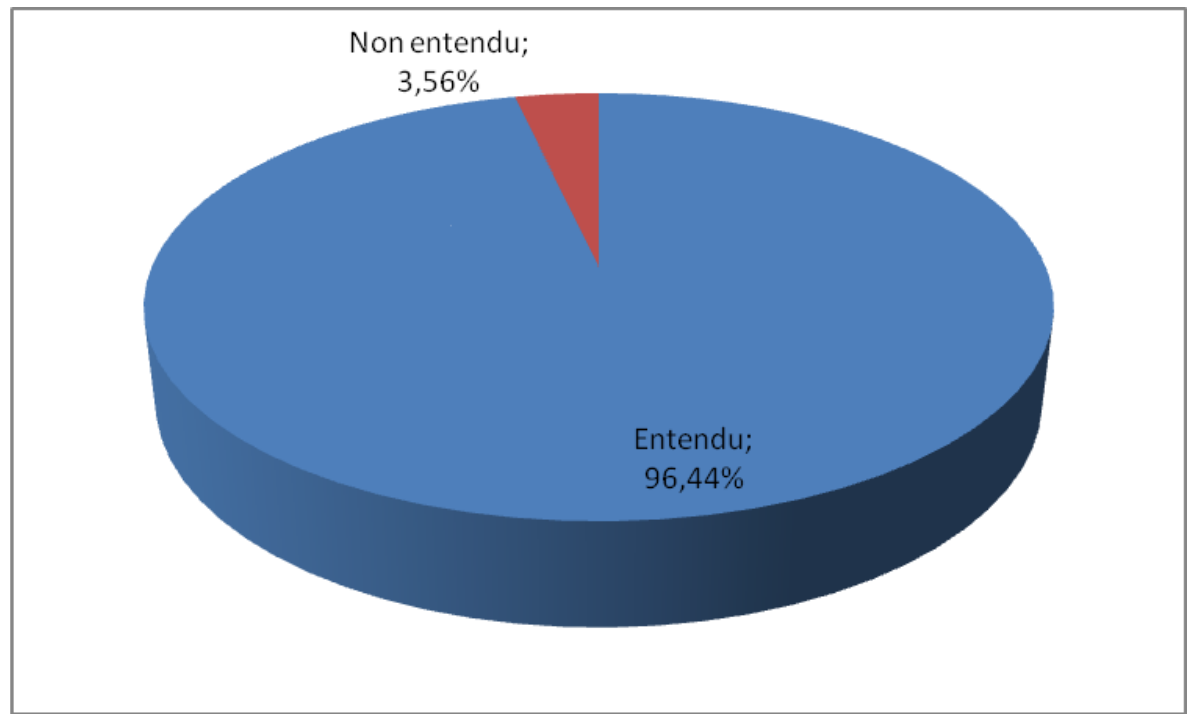

Figure 6: Connaissance à propos T. articulata

Figure 6: Knowledge about $T$. articulata

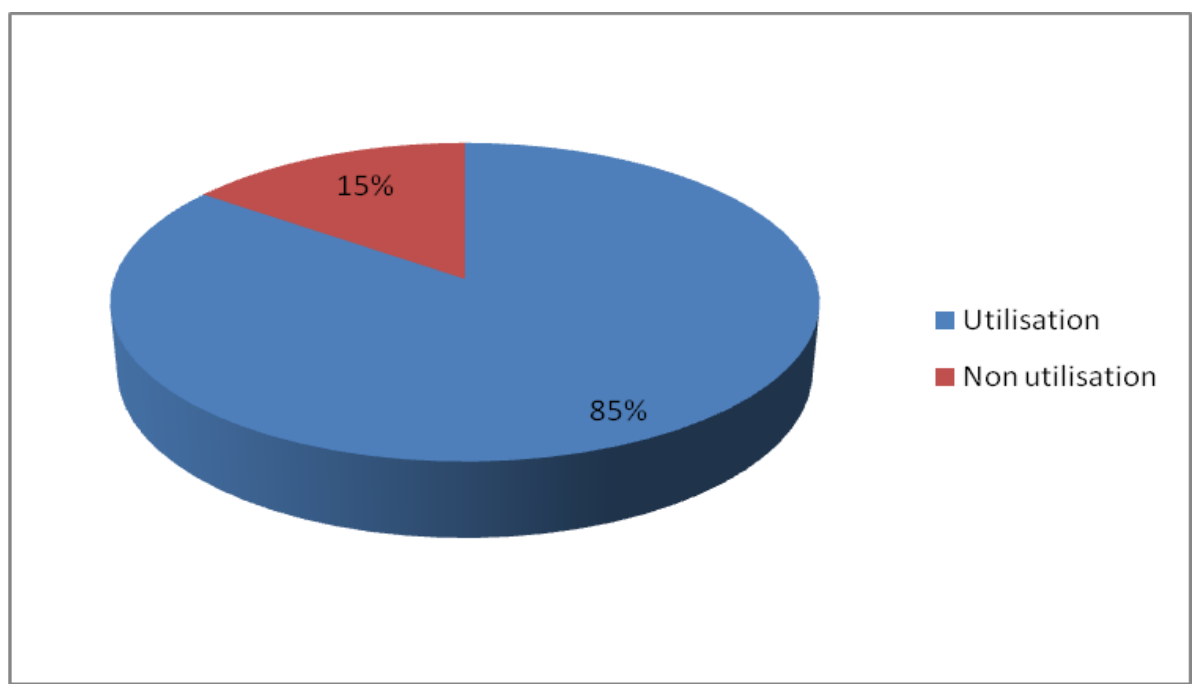

Figure 7: Utilisation de T. articulata

Figure 7: Use of T. articulata 


\section{Domaines d'utilisation}

Les informations recueillies sur les domaines d'utilisation du $T$. articulata ont révélé qu'un taux de $29,70 \%$ d'usage est thérapeutique suivi par l'usage cosmétique et industriel, soit $19,61 \%$ et $18,05 \%$ respectivement. Tandis que le pâturage vient au dernier rang avec 6,50\% (Fig. 8).

\section{Pathologies traitées par $T$. articulata}

Les domaines d'utilisation de $T$. articulata dans la thérapie sont variables selon le cas de la maladie traitée. Ainsi, les affections gastro-intestinales, la fièvre et les maux de tête présentent $26,69 \%$, $25,05 \%$ et $21,07 \%$, respectivement. Tandis que les affections articulaires et uro-génitales ainsi que les affections cardio-vasculaires viennent au dernier rang, soit $1,40 \%$ contre $0,70 \%$ (Fig. 9).

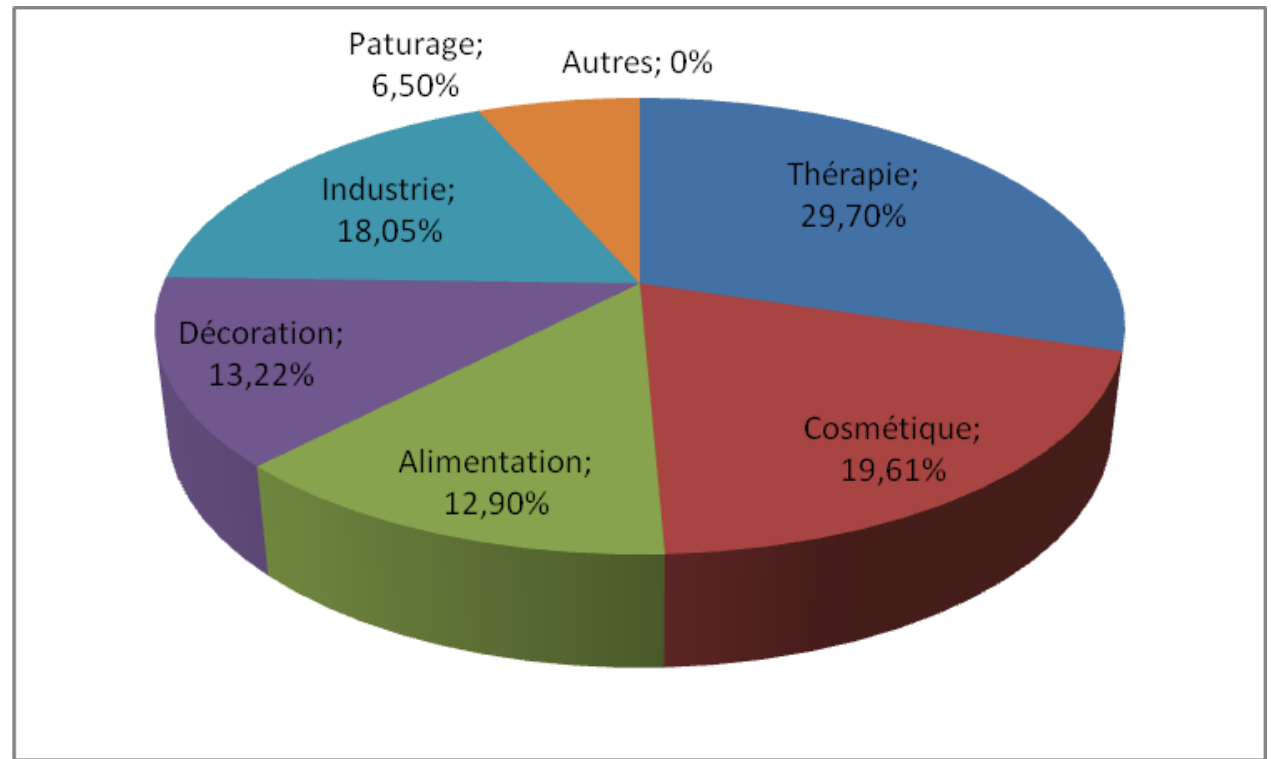

Figure 8: Domaines d'utilisations de T. articulata

Figure 8: Areas of use of T. articulata

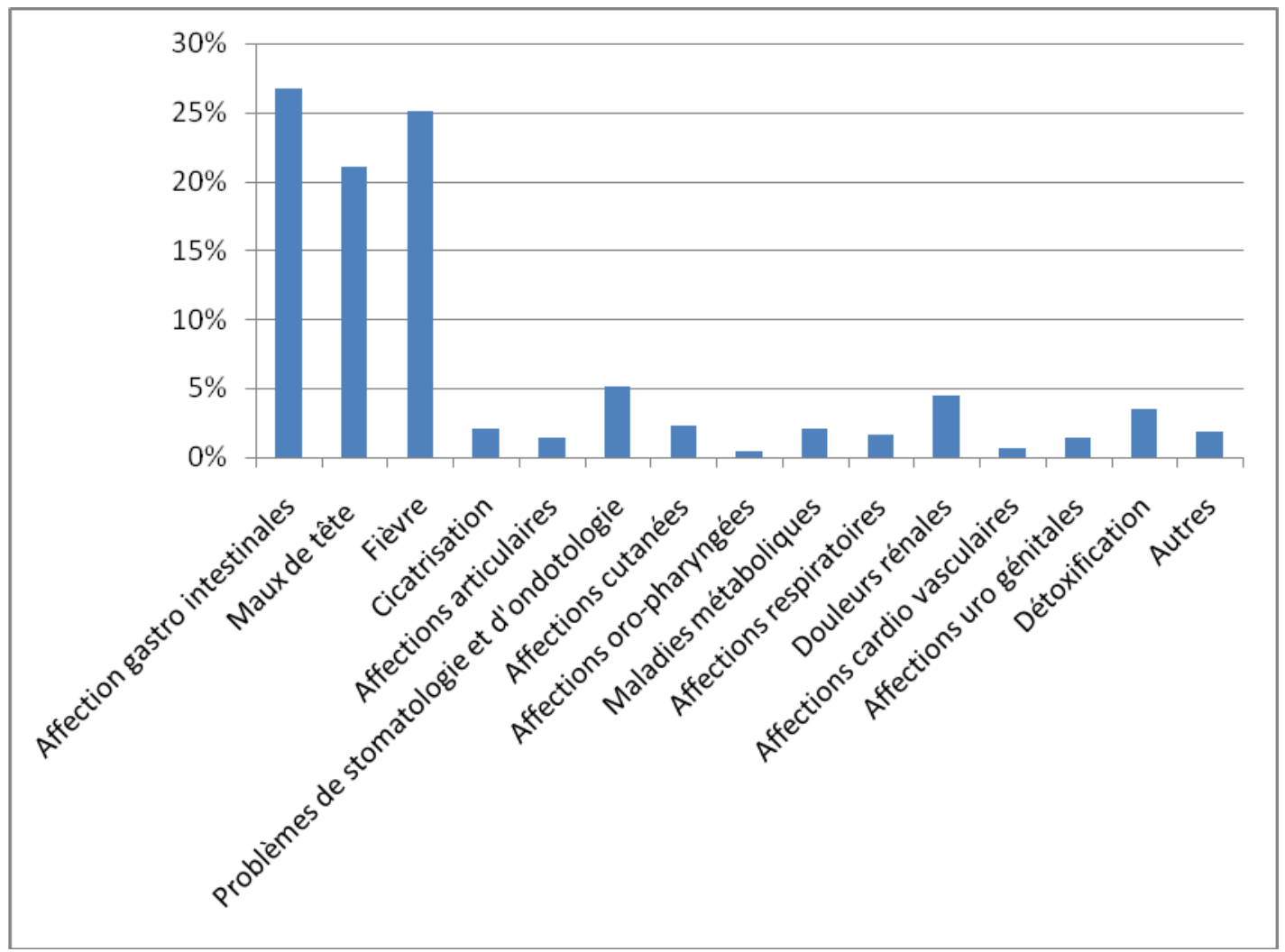

Figure 9: Pathologies traitées par T. articulata

Figure 9: Pathologies treated by $T$. articulata 
Antérieurement, il a été rapporté, qu'en cataplasme, les feuilles du thuya de barbarie sont appliquées sur les trempes contre les céphalées et la fièvre dans la phytothérapie traditionnelle dans le district de BéniMellal (El Azzouzi \& Zidane 2015). En outre, il a été mis en évidence dans le Parc National de Talassemtane (Rif occidental du Maroc) que la même plante est également utilisée pour traiter les maux de tête (Rhattas et al. 2016). Tandis que l'étude menée par Fakchich \& Elachouri (2014), a rapporté que $T$. articulata est employée pour traiter les problèmes digestifs dans la région orientale marocaine, ce qui rejoint amplement nos résultats.

II est à mentionner que d'autres maladies ont été traitées par les utilisateurs du thuya de barbarie à savoir: l'allergie en mélangeant l'eau avec les grains de cette plante, l'inflammation des yeux et le vertige. Utilisation en cosmétique

En cosmétique, la lotion(rhasoul) présente $32 \%$ des utilisations du thuya de barbarie tandis que le goudron végétale à base de la dite plante ne présente que 12\% (Fig. 10).

Quelque soit son utilisation, il a été rapporté, auparavant, que $T$. articulata en poudre, est employée dans les soins des cheveux dans le district de Béni Mellal (El Azzouzi \& Zidane 2015) et en association avec le Henné dans la région de Zaër (Lahsissene et al. 2009).

Par ailleurs, d'autres utilisations ont été notées par les enquêtés à savoir l'emploi hygiénique de l'écorce de $T$. articulata pour nettoyer les aisselles.

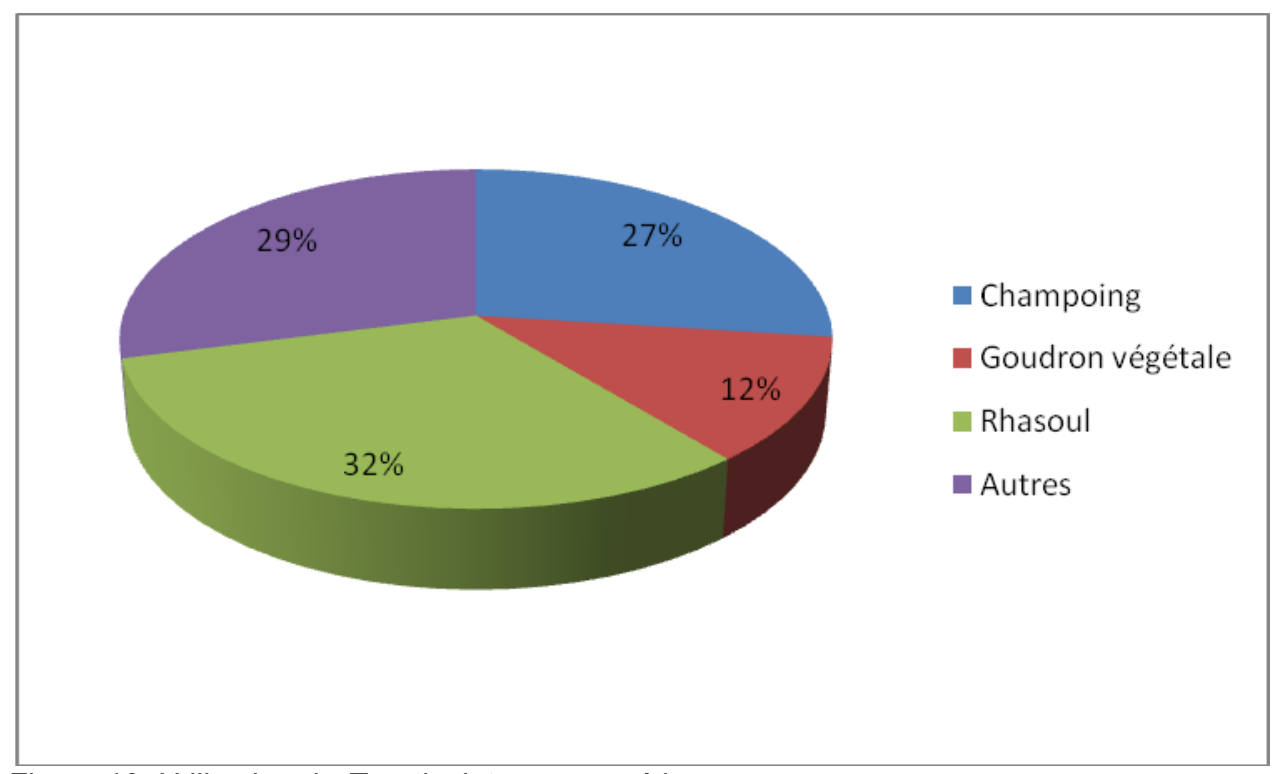

Figure 10: Utilisation de $T$. articulata en cosmétique

Figure 10: Use of $T$. articulata in cosmetics

\section{Utilisation en alimentation}

En ce qui concerne l'utilisation en alimentation, le questionnaire a révélé que les habitants de la région utilisent la plante $T$. articulata comme un aromatisant de petit lait, soit $84,82 \%$. A l'encontre, $15,18 \%$ qui reste représente la catégorie utilisée de la dite plante sous d'autres formes, telles que le mélange des feuilles de thuya avec le miel (Fig. 11).

\section{Utilisation en industrie}

En industrie, la menuiserie représente $47,69 \%$ des utilisations du thuya de barbarie tandis que les utilisations dans les domaines d'exportation de bois, de la tannerie et la papeterie sont estimées á des pourcentages de $18,71 \% ; 15,78 \%$ et $11,11 \%$ respectivement (Fig. 12). Le reste (3,50\%) correspond à d'autres utilisations indiquées par les enquêtés à savoir l'emploi de bois du thuya de barbarie dans le secteur artisanal pour la production des tables, boites, œuvres d'arts, toits de maisons ainsi que l'utilisation de la gomme sandaraque issue de la résine de $T$. articulata pour préparer le vernis et la peinture comme il a été rapporté par Rhattas et al. (2016).

\section{Parties de la plante utilisées en thérapie}

Dans la zone d'étude, les feuilles de $T$. articulata sont les parties les plus utilisées dans la thérapie avec un pourcentage de $70,80 \%$, suivies par les rameaux qui représentent $9,82 \%$, puis par les fleurs avec un pourcentage d'utilisation de 4,13\% (Fig. 13).

\section{Parties de la plante utilisées en cosmétique}

La Fig. 14 démontre que la majorité des personnes utilisent les feuilles à un pourcentage de $66,03 \%$, puis les rameaux à $13,20 \%$ et les tiges à $5,28 \%$ en cosmétique. 


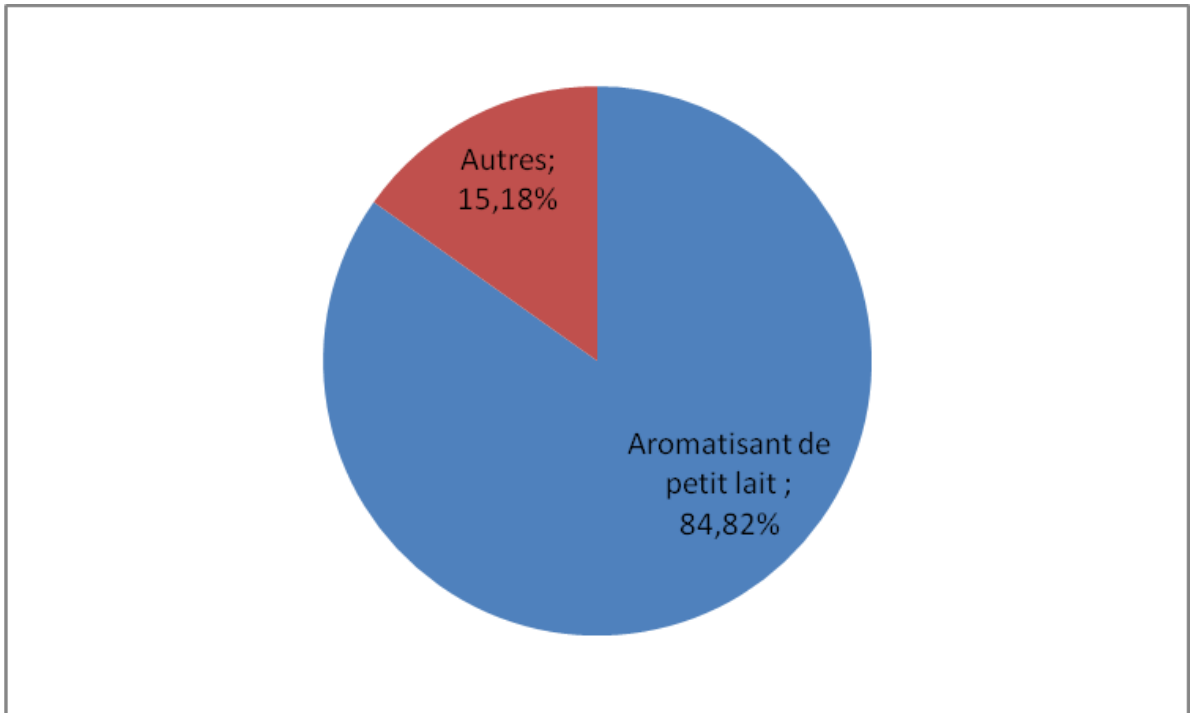

Figure 11: Utilisation de $T$. articulata en alimentation

Figure 11: Use of $T$. articulata in food

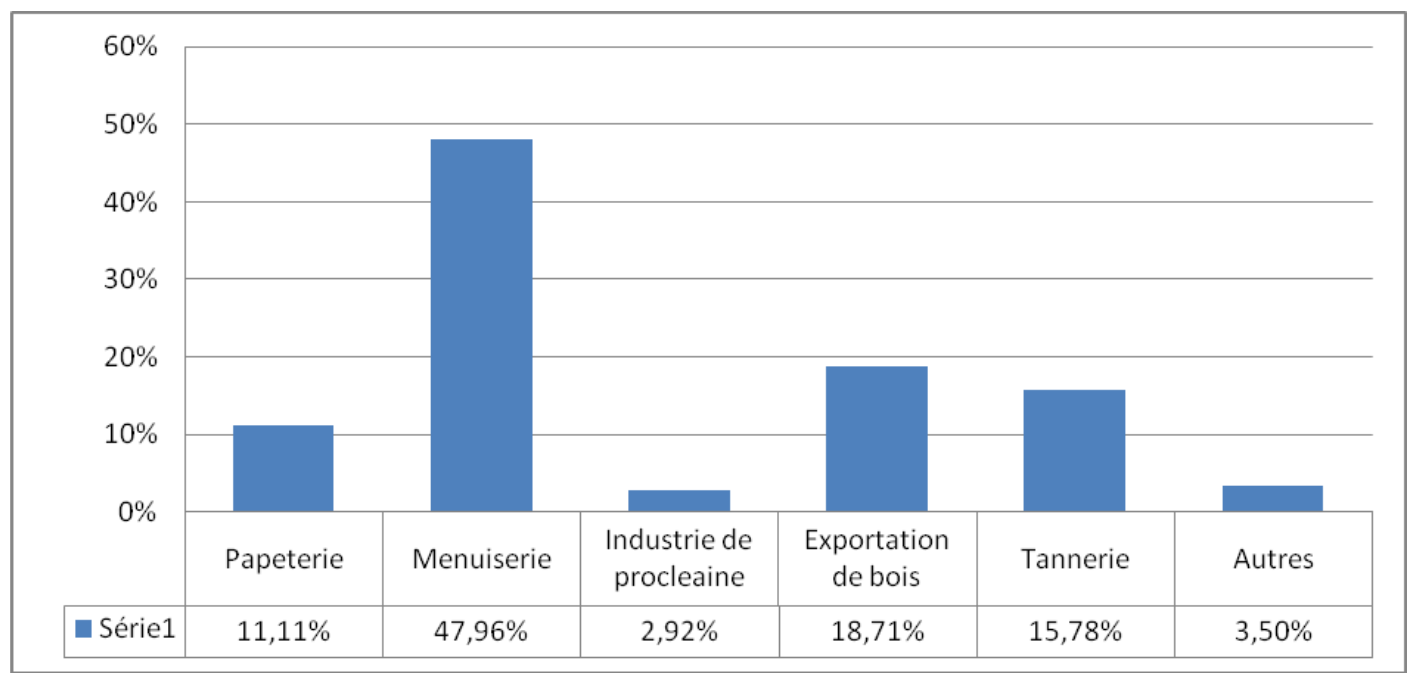

Figure 12: Utilisation de T. articulata en industrie

Figure 12: Use of $T$. articulata in industry

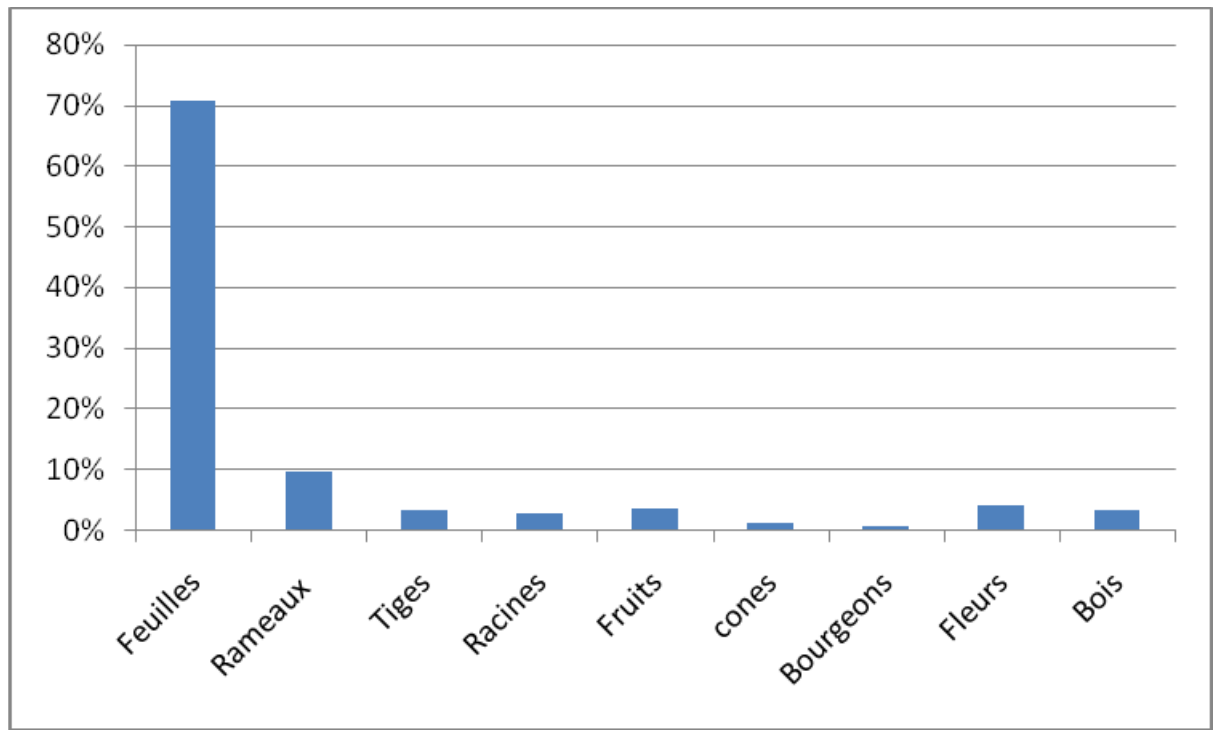

Figure 13: Parties utilisées de T. articulata en thérapie

Figure 13: Parts used of T. articulata in therapy 


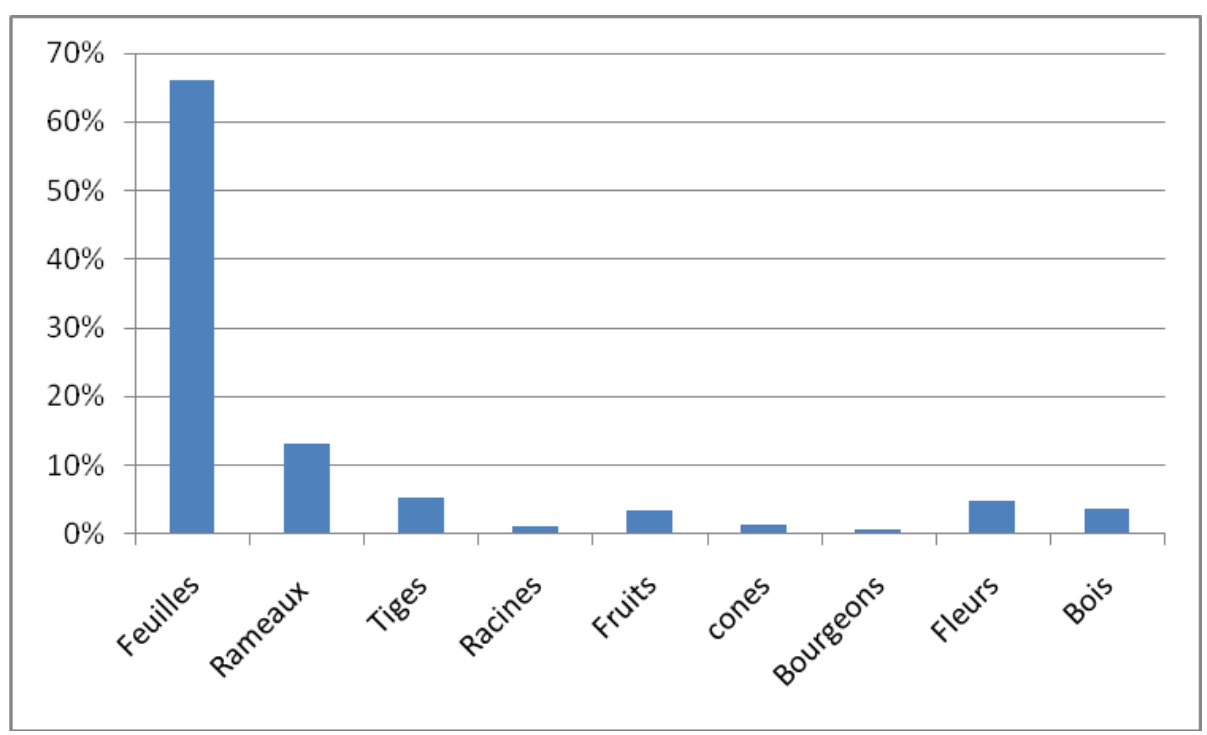

Figure 14: Parties utilisées de T. articulata en cosmétique

Figure 14: Used parts of $T$. articulata in cosmetics

Parties de la plante utilisées en alimentation

Dans la zone d'étude, on déduit que les parties les plus utilisées en alimentation sont les feuilles qui représentent un pourcentage de $67,21 \%$, suivies par les rameaux à un pourcentage de $13,11 \%$ et le reste des parties présente 19,68\%(Fig. 15).

Selon la présente étude, ce sont les feuilles qui sont les plus utilisées dans les trois domaines investigués. Ceci peut être expliqué par le fait que les feuilles sont le siège de la photosynthèse, le réservoir de matières organiques qui en découlent et qui peuvent être responsables des propriétés biologiques de la plante (Rhattas et al. 2016, Chraibi et al. 2018, Zahir et al. 2018). De plus, la fréquence d'utilisation élevée de feuilles peut être expliquée par l'aisance et la rapidité de leur récolte (Bammou et al. 2015, Rhattas et al. 2016). Ce résultat est consistant avec celui de plusieurs recherches ethnobotaniques préalables (Azzi et al. 2012, El Yahyaoui et al. 2015, Ghourri et al. 2012, Fakchich \& Elachouri 2014, Bammou et al. 2015, Rhattas et al. 2016, Ben Akka et al. 2017, Katiri et al. 2017, Kadri et al. 2018, Chraibi et al. 2018, Salhi et al. 2019).

\section{Forme d'utilisation de la plante}

La Fig. 16 indique que la forme d'emploi la plus utilisée du thuya de barbarie est la forme séchée à un pourcentage de $76,70 \%$, contre la forme fraiche à un pourcentage de $23,30 \%$.

Ces résultats sont concordants avec ceux de l'étude menée par Salhi et al. (2019) qui a rapporté que la forme sèche est la plus utilisée pour le traitement des brûlures de la peau, par les plantes médicinales, dans la région de Rabat (Maroc), soit $79,28 \%$ contre $23,57 \%$ pour l'utilisation de la forme fraîche.
Incontestablement, il a été rapporté par une autre investigation que le séchage qui se fait à l'abri de la lumière permet la préservation de la majorité des principes actifs des plantes (Chraibi et al. 2018).

\section{Forme d'emploi de la plante}

La Fig. 17 démontre que la plupart des enquêtés utilisent la dite plante sous forme de poudre à un pourcentage de $70,50 \%$, puis sous forme d'huile essentielle (HE) et d'extrait (avec des pourcentages respectifs de $10,91 \%$ et $10,32 \%$ ). Ce constat corrobore le fait que l'emploi des plantes sous forme de poudre est la forme la plus utilisée dans de nombreuses enquêtes ethnopharmacologiques(El Yahyaoui et al. 2015, Ghourri et al. 2012, Salhi et al. 2019). En revanche, dans autres études, ce sont plutôt d'autres formes qui sont plus employées à savoir tisane (Bammou et al. 2015, Chraibi et al. 2018).

\section{Mode de préparation à base de $T$. articulata en thérapie}

Le mode de préparation le plus utilisé du thuya de barbarie en thérapie est la macération à un pourcentage de $60,34 \%$, suivie par l'infusion avec un pourcentage de $13,99 \%$ puis le cataplasme qui représente $11,35 \%$ (Fig. 18). Ce résultat est conforme à celui de l'étude ethnobotanique réalisée par Azzi et al. (2012) qui ont trouvé que la macération des feuilles de $T$. articulata est employée pour le traitement de diabète dans le nord-ouest et le sud-ouest d'Algérie.

Par ailleurs, d'autres modes de préparation représentant $8,45 \%$ ont été indiqués telle que la fumigation de la poudre de thuya. 


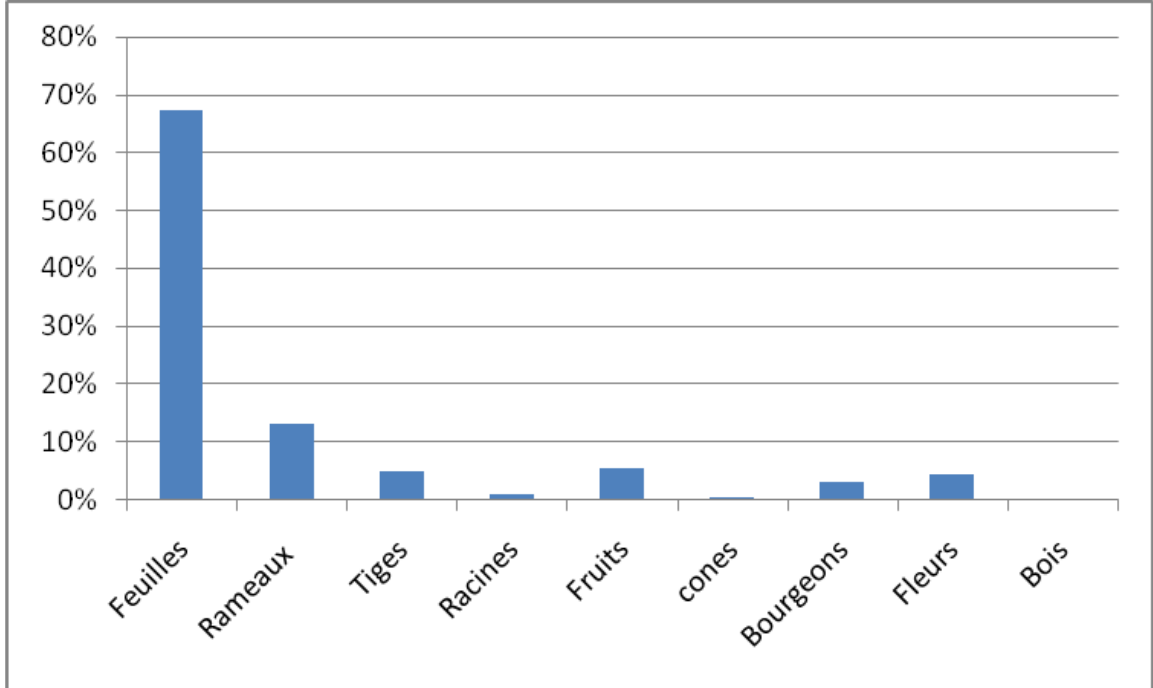

Figure 15: Parties utilisées de T. articulata en alimentation

Figure 15: Used parts of $T$. articulata in food

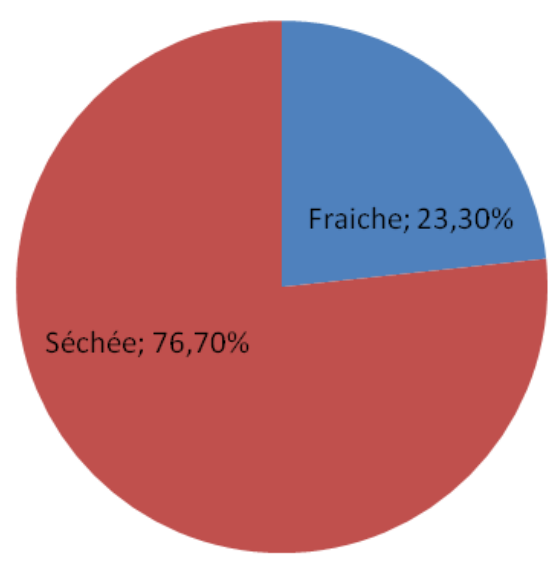

Figure 16: Forme d'utilisation de $T$. articulata

Figure 16: Form of use of $T$. articulata

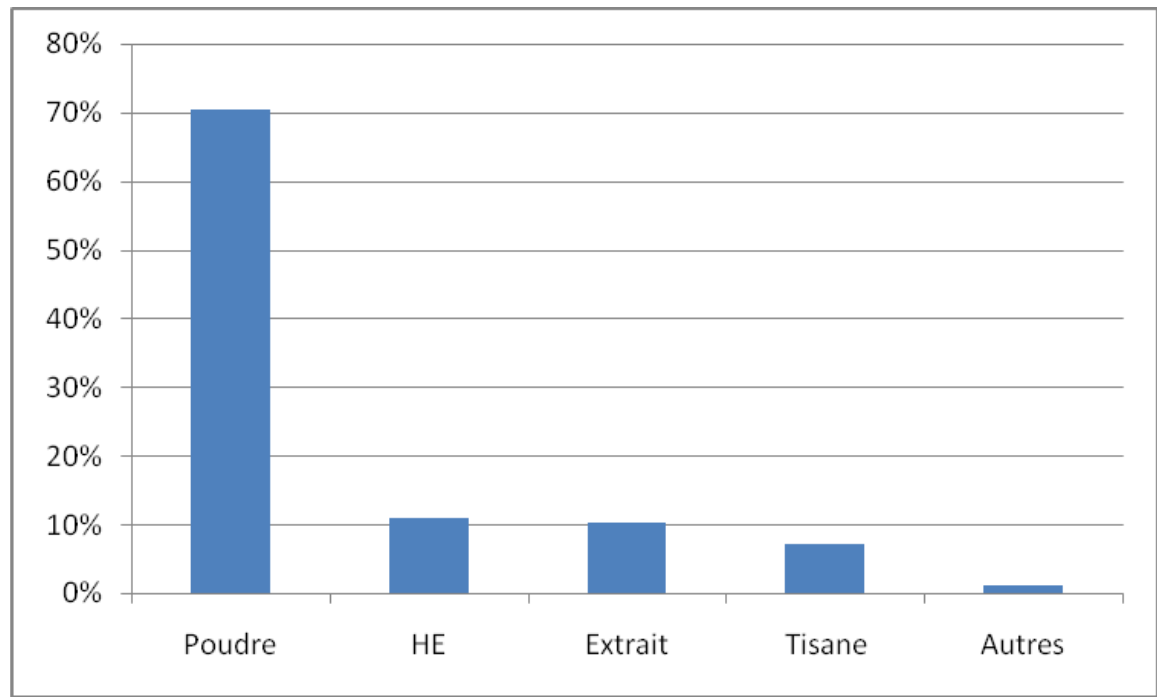

Figure 17: Forme de manipulation de $T$. articulata. HE: huile essentielle Figure 17: Form of manipulation of $T$. articulata. HE: essential oil 


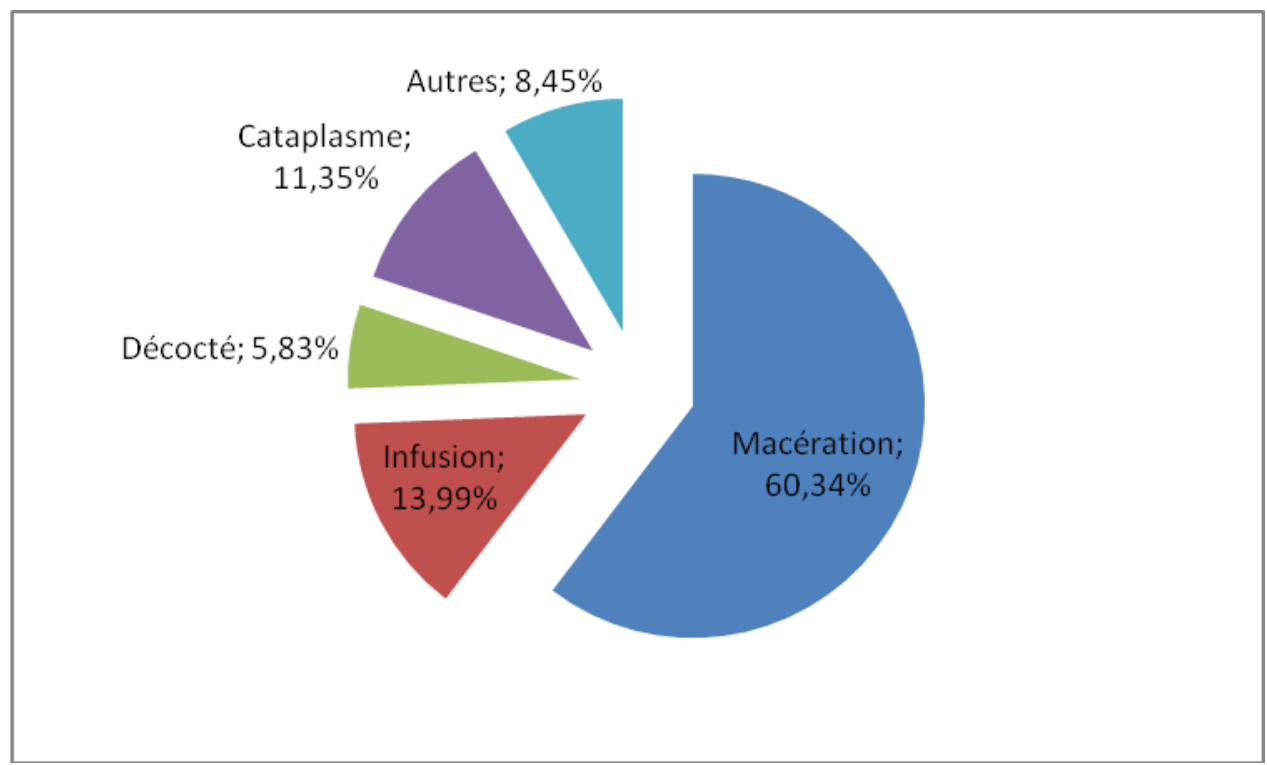

Figure 18: Mode de préparation à la base de $T$. articulata en thérapie

Figure 18: Method of preparation at the base of $T$. articulata in therapy

Mode de préparation à base de $T$. articulata en cosmétique

Parmi les modes de préparation les plus utilisés en cosmétique, on trouve que la macération représente un pourcentage important de $60,66 \%$, puis l'infusion à $10,42 \%$ et vient ensuite le décocté à $9,95 \%$ (Fig. 19).

Mode de préparation à base de $T$. articulata en alimentation

D'après notre enquête, on constate que la plupart des enquêtés utilisent le mode de macération en alimentation $(55,03 \%)$, suivi par le mode d'infusion, soit $14,09 \%$ (Fig. 20).

Néanmoins, ces résultats ne sont pas similaires à ceux trouvés par l'étude menée par Kadri et al. (2018) mettant en évidence que la méthode de préparation la plus répandue de 46 plantes médicinales dans une région hyper aride du Sudouest Algérien est la décoction avec un pourcentage de $51.17 \%$. D'une manière analogue, le même constat a été signalé par autres investigations (Fakchich \& Elachouri 2014, Bachiri et al. 2015, Katiri et al. 2017, Chraibi et al. 2018). D'une manière générale, la meilleure utilisation d'une plante serait celle qui en préserverait toutes les propriétés tout en permettant l'extraction et l'assimilation des principes actifs (Rhattas et al. 2016).

\section{Voie d'administration de la plante en thérapie}

En thérapie, 58,53\% des enquêtés administrent les préparations à la base de $T$. articulata oralement, tandis que $31,98 \%$ seulement utilisent la voie cutanée, contre 9,48\% emploient autres voies d'administration comme la voie nasale et urogénitale (Fig. 21).
Ce résultat est identique à celui révélé par Fakchich \& Elachouri (2014) démontrant que le mode d'administration le plus sollicité des préparations à base de $T$. articulata est la voie orale.

\section{Dose utilisée}

Selon les enquêtés, l'utilisation de $T$. articulata est dosée par plusieurs méthodes dont les majoritaires sont présentées notamment par une cuillère et une dose précise, soit $37,20 \%$ et $34,88 \%$ respectivement. Alors que le dosage par une poignée vient au troisième rang avec $22,67 \%$ (Fig. 22). Ainsi, $59,87 \%$ des personnes interrogées emploient la plante en question sans dose précise. Ce résultat est proche de celui obtenu par d'autres études pour lesquelles les plantes médicinales sont utilisées avec des doses approximatives sans aucune précision (Azzi et al. 2012, Bammou et al. 2015, Chraibi et al. 2018, Kadri et al. 2018). Or, le surdosage pourrait provoquer des effets néfastes sur la santé car il y a souvent une toxicité dosedépendante (Chraibi et al. 2018). Indubitablement, les plantes médicinales ont des effets indésirables quand elles sont utilisées de façon incorrecte par les patients (Rhattas et al. 2016). A l'inverse une dose insuffisante pourrait mener à une inefficacité de l'effet recherché (Bammou et al. 2015).

\section{Nombre de prise par jour}

Dans la zone étudiée, on constate que la majorité des enquêtés utilisent $T$. articulata une seule fois par jour avec un pourcentage de $59,88 \%$, alors que seul $26,64 \%$ l'utilisent deux fois par jour. Par contre, $8,38 \%$ des questionnés emploient leur préparation végétale trois fois par jour (Fig. 23). Par ailleurs, le nombre de prise diffère d'une plante à une autre. Effectivement, il a été souligné par Bammou et 
al.(2015) que Pistacia lentiscus a été administrée deux fois par jour dans $44 \%$ des cas. D'autres périodes d'utilisation ont ét é mentionnées comme l'emploi du thuya pendant la période de la maladie ou deux fois/semaine, une heure/jour, voire trois fois/jour.

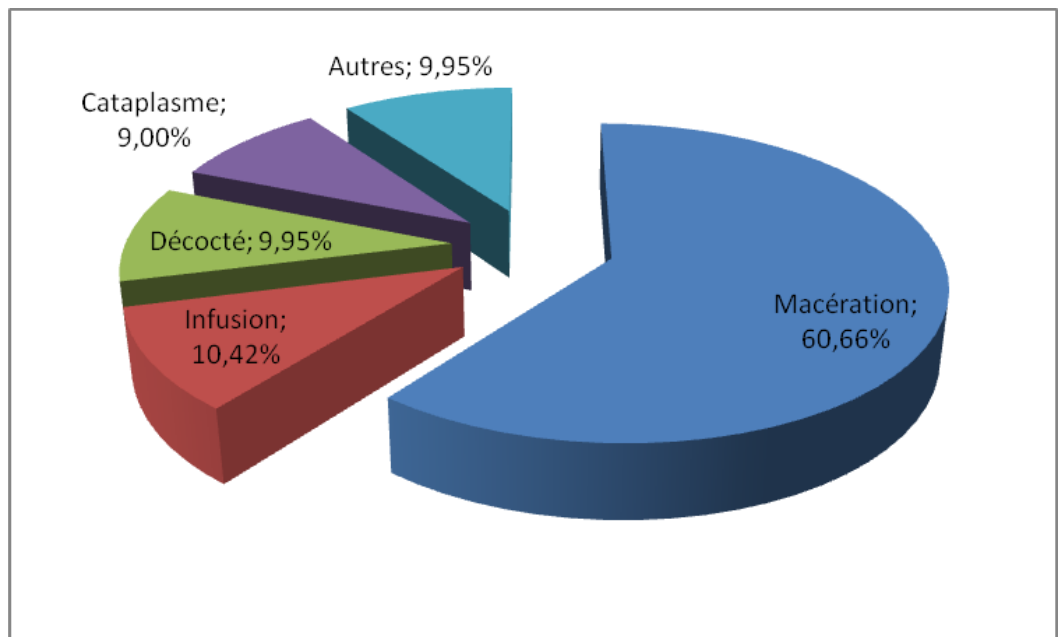

Figure 19: Mode de préparation à la base de T. articulata en cosmétique Figure 19: Method of preparation at the base of $T$. articulata in cosmetics

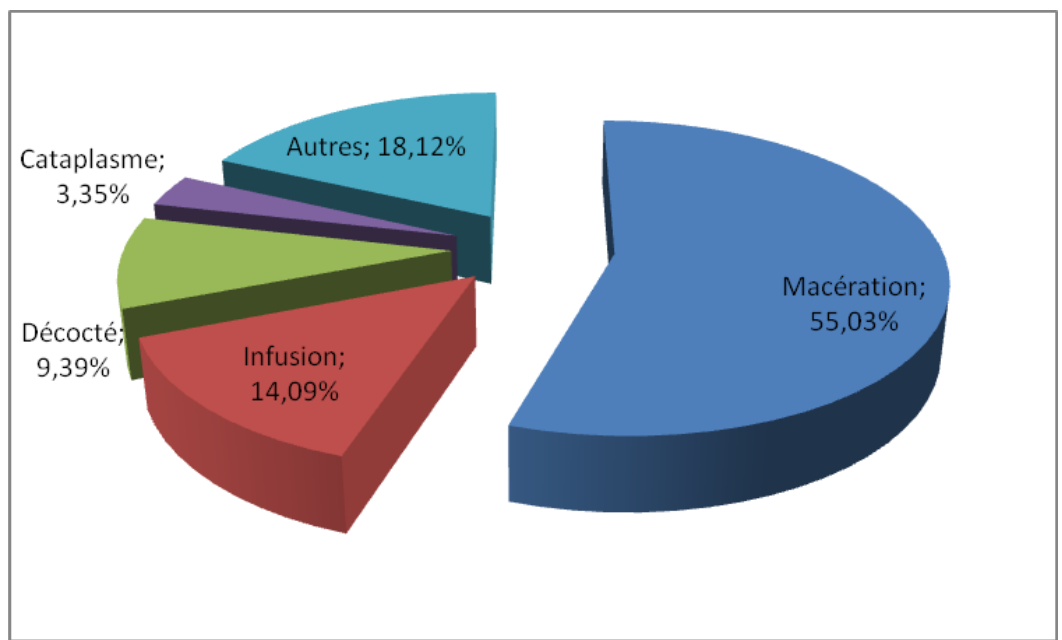

Figure 20: Mode de préparation à la base de $T$. articulata en alimentation Figure 20: Method of preparation at the base of $T$. articulata in food

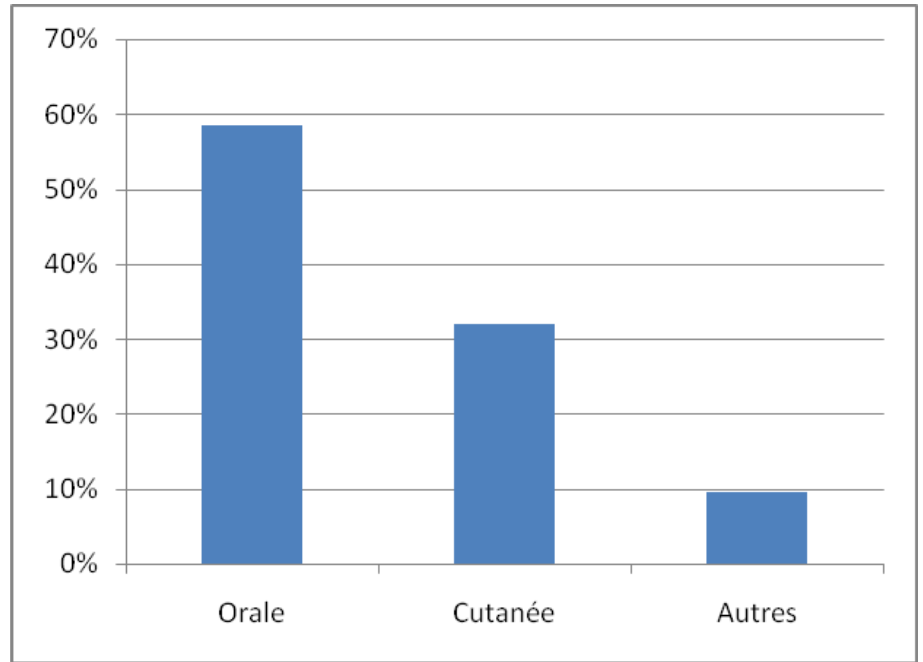

Figure 21: Voies d'administration de $T$. articulata en thérapie Figure 21: Administration ways of $T$. articulata in therapy 


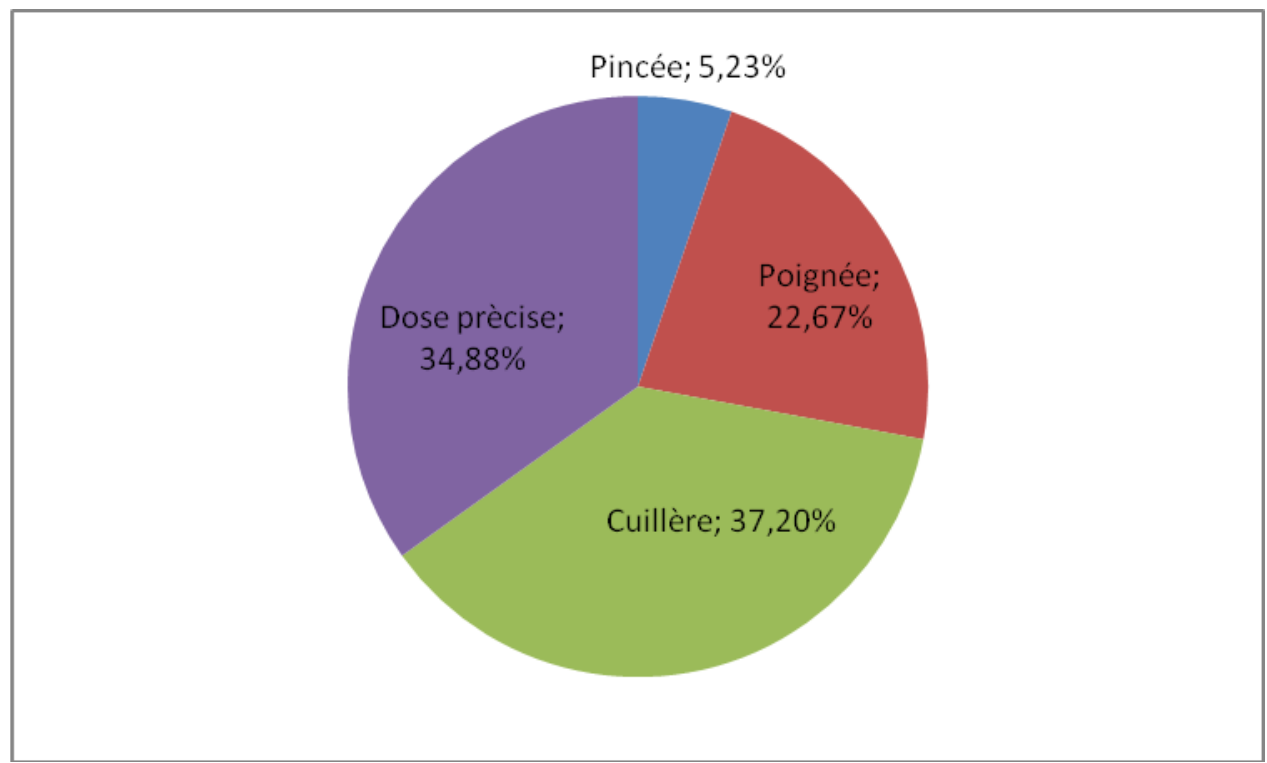

Figure 22: Dose utilisée de $T$. articulata par les enquêtés Figure 22: Dose used of $T$. articulata by the respondents

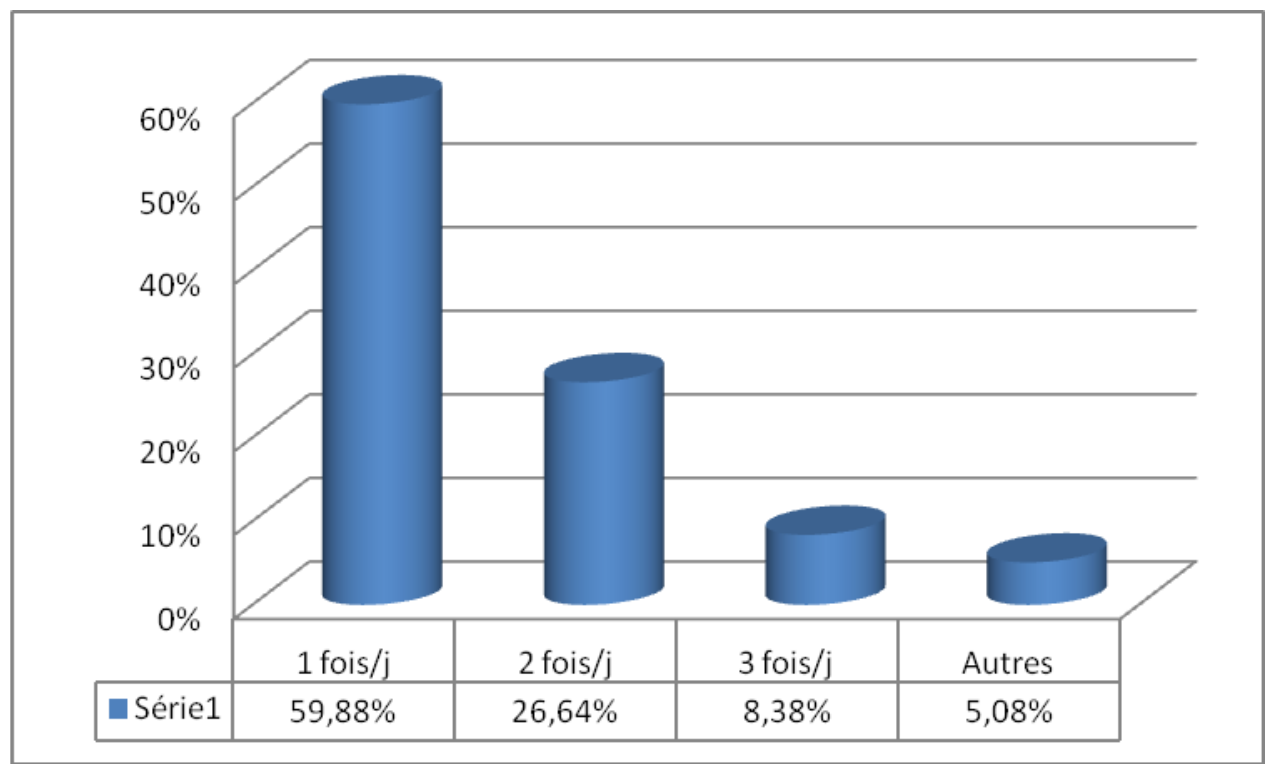

Figure 23: Nombre de prise par jour des préparations à la base de la plante $T$. articulata Figure 23: Number of doses per day of preparations based on T. articulata

Période d'utilisation de $T$. articulata pour traiter la fièvre, les maux de tête et les affections gastrointestinales

D'après la Fig. 24, on constate que:

- $\quad$ Entre sept à vingt deux enquêtés utilisent $T$. articulata pour traiter les maux de tête pendant un jour jusqu'à trois jours. Les autres enquêtés l'emploient selon la période durant laquelle ils souffrent de la maladie.

- $\quad$ Entre sept à dix huit personnes utilisent $T$. articulata comme remède pour éviter les affections gastro-intestinales pendant une période comprise entre 1 jour jusqu'à 7 jours.
- $\quad$ Entre six à seize personnes utilisent le thuya de barbarie pendant 1 jour jusqu'à 3 jours pour traiter la fièvre, tandis que d'autres l'emploient pendant une courte période allant du 30 min à 1 heure.

Un cas particulier a été indiqué par les enquêtés à propos du traitement de la fièvre ou la prévention des frappes de chaleur au cours de l'été chez les enfants. Ainsi, les parents ont soumis leurs enfants de six mois voire des nouveaux nés à des cataplasmes ou les ont trempés dans des bains à base des préparations des feuilles de thuya de barbarie pendant presque 15 à 30 minutes. Le lavage des corps d'enfants ne se fait qu'après deux heures d'exposition à cette préparation. 
Période d'utilisation de $T$. articulata sous forme de shampoing, goudron végétal et de lotion Selon les résultats de notre enquête, vingt et une personnes utilisent $T$. articulata sous forme de shampoing pendant 1 jour; tandis qu'un faible nombre des personnes l'emploient sous forme de goudron végétal et de lotion durant des périodes comprises entre 30 min jusqu'à 3 jours et entre 1 jour jusqu'à 7 jours d'utilisation, respectivement (Fig. 25).

\section{Evaluation du résultat obtenu après l'emploi de} $T$. articulata en thérapie

D'après notre enquête, $94,07 \%$ des personnes sont satisfaits des résultats obtenus après l'utilisation des préparations à base de thuya de barbarie en thérapie, contre $5,93 \%$ qui ont en été insatisfaits dont $2,6 \%$ jugent néfaste le résultat acquis après traitement (Fig. 26).

\section{Evaluation du résultat obtenu après l'emploi de $T$. articulata en cosmétique}

Les résultats d'utilisation du thuya en cosmétique révèlent que $94,82 \%$ des personnes enquêtées sont satisfaites contre $5,16 \%$ des gens sont insatisfaits dont $3,44 \%$ trouvent l'emploi de cette essence végétale ne porte aucun résultat (Fig. 27).

Ces résultats sont en accord avec ceux de Chraibi et al. (2018) ayant rapporté que presque la totalité des personnes enquêtées sont satisfaites des résultats obtenus après utilisation de trois plantes médicinales (Mentha pulegium, Mentha piperita et Pelargonium graveolens) dans la région de Taounate au Maroc, soit $93 \%$. Alors que $7 \%$ de la population locale croient que les plantes étudiées ont des effets secondaires.

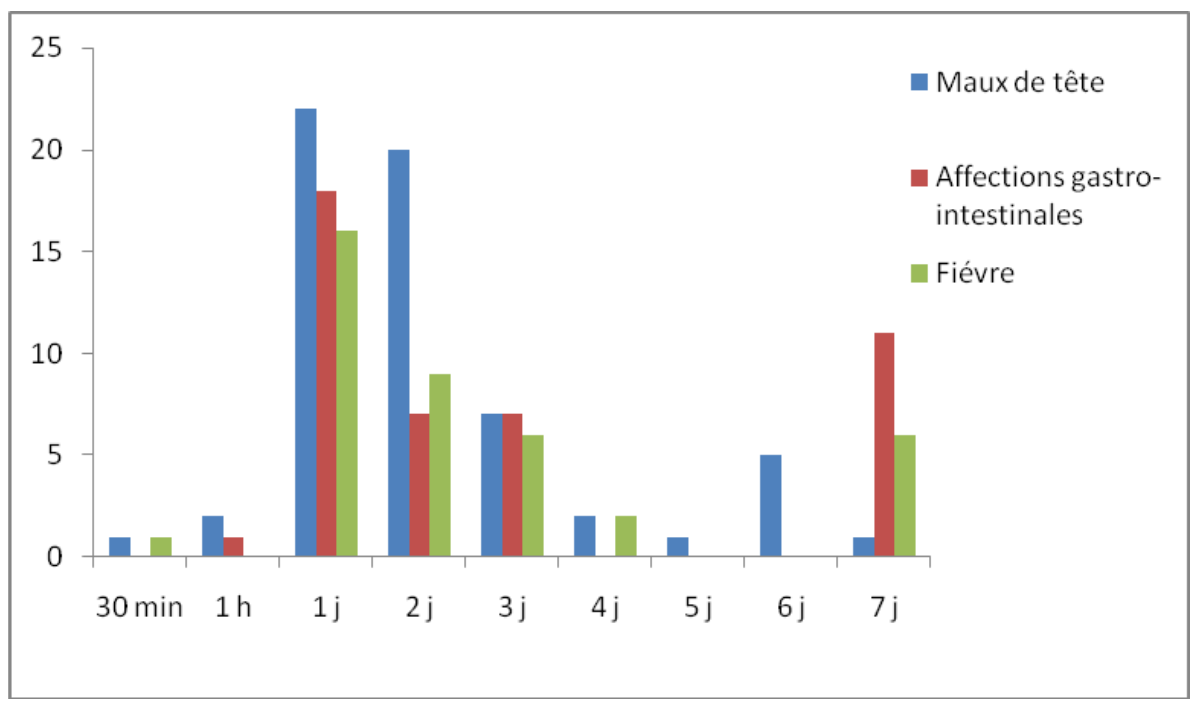

Figure 24: Période d'utilisation de T. articulata pour traiter les maux de tête, les affections gastro-intestinales et la fièvre

Figure 24: Period of use of $T$. articulata to treat headaches, gastrointestinal complaints and fever

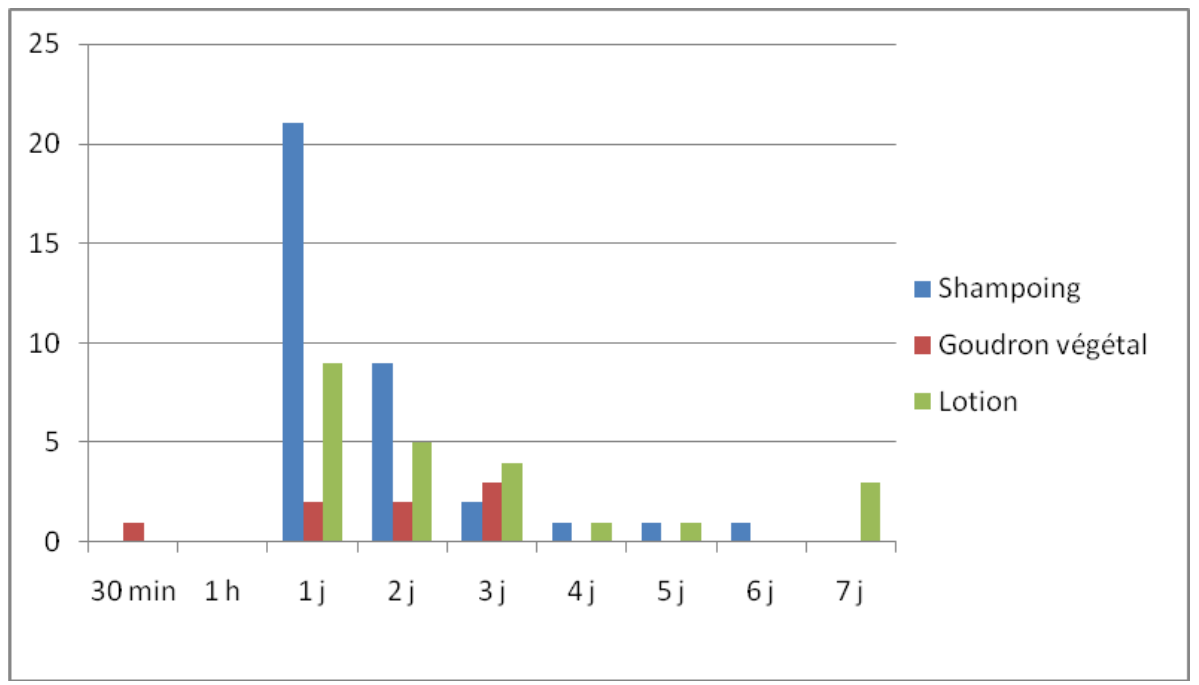

Figure 25: Période d'utilisation de T. articulata sous forme de shampoing, goudron végétal et de lotion Figure 25: Period of use of $T$. articulata in the form of shampoing, vegetable tar and lotion 


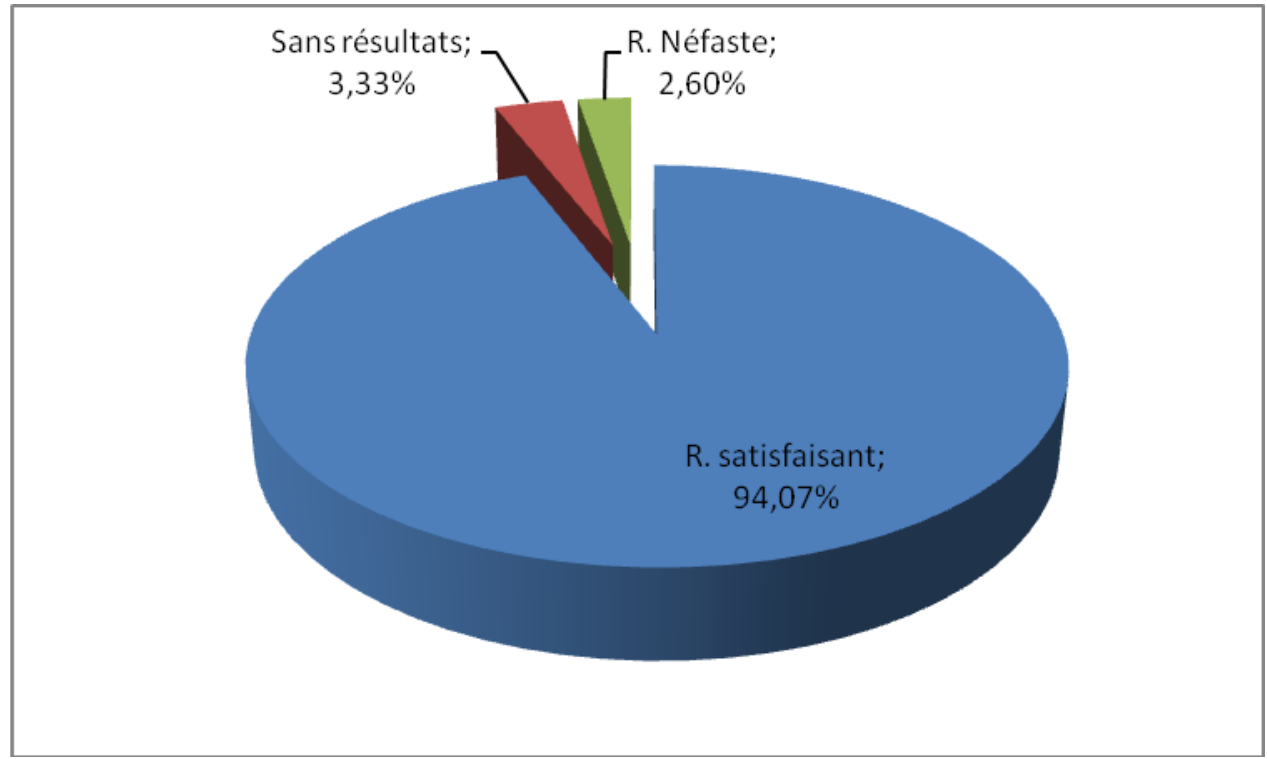

Figure 26: Evaluation des résultats obtenus après l'emploi de $T$. articulata en thérapie Figure 26: Evaluation of the results obtained after the use of $T$. articulata in therapy

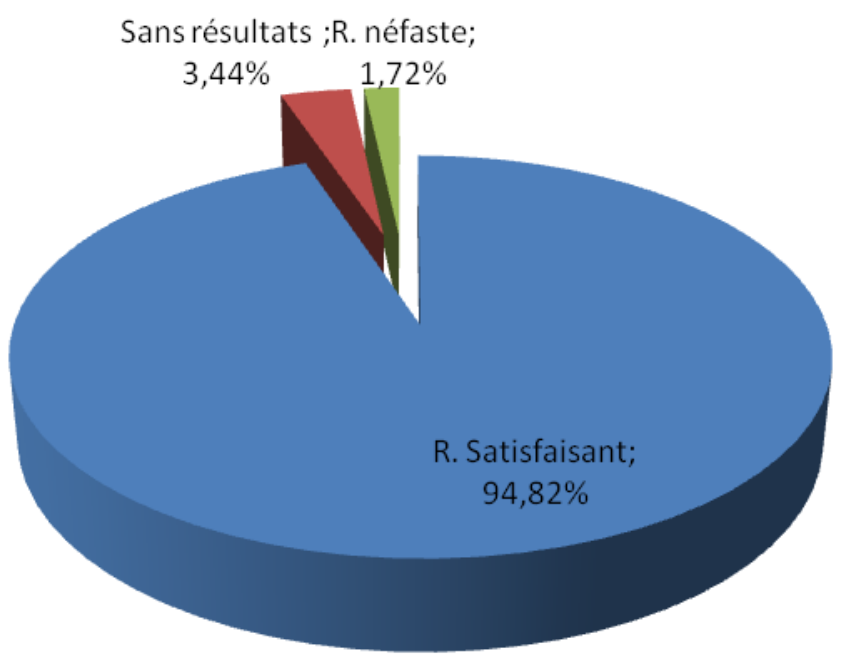

Figure 27: Evaluation des résultats obtenus après l'emploi de $T$. articulata en cosmétique Figure 27: Evaluation of the results obtained after the use of $T$. articulata in cosmetics

\section{Toxicité}

La plupart des enquêtés (soit $86,33 \%$ ) jugent que le thuya de barbarie n'est pas toxique, contre $13,67 \%$ des gens qui la considèrent comme toxique (Fig. 28).

Ce résultat rejoint celui rapporté par plusieurs investigations concluant que le thuya de barbarie n'est pas toxique. Incontestablement, dans l'étude menée par Jemli et al. (2016), il a été indiqué qu'il n'y avait pas de changements visibles dans le comportement général et le poids des souris du groupe traité avec une dose de $2 \mathrm{~g} / \mathrm{kg}$ par rapport au groupe témoin après l'administration de l'huile essentielle(HE) de $T$. articulata pendant 15 jours. II a été conclu, consécutivement, que l'administration orale de l'HE n'a eu aucun effet sur la croissance et les fonctions des animaux à cette concentration. D'autre part, l'administration d'HE à différentes doses (2 et $5 \mathrm{~g} / \mathrm{kg}$ ) n'a entraîné aucune mortalité.

En outre, dans une autre investigation de toxicité aiguë, I'HE de thuya issu de la forêt Tafoughalt Zegzell marocaine a été délivrée par inhalation à des doses de $1 \%$ et $3 \%$ aux rats. Après une surveillance pendant $24 \mathrm{~h}$, aucun de décès lié au traitement n'a été enregistré et aucun signe de toxicité n'a été développé que ce soit chez les animaux traités ou les témoins tout au long de l'étude. De plus, il n'y a eu aucun changement de comportement et aucun signe anormal n'a été observé (Sadiki et al. 2018). 
Cependant, la plupart des tests ne mesurent que la toxicité aiguë. Or, la toxicité est un terme relatif et varie selon la partie de la plante extraite et les quantités consommées en fonction du temps. Par conséquent, il est essentiel de tester la toxicité de l'extrait brut, de l'HE et des composés individuels afin de recueillir plus d'informations sur les réactions indésirables éventuelles pouvant résulter d'une utilisation du thuya à long terme (Azzi et al. 2012).

De plus, de nombreuses plantes peuvent être dangereuses car elles contiennent à la fois des substances bénéfiques et toxiques. Ainsi, les préparations traditionnelles sont des mélanges complexes de substances inconnues (Azzi et al. 2012). Devant cette situation, il nécessaire de réaliser des études toxicologiques et cliniques des plantes les plus utilisées par la population comme le cas de $T$. articulata afin de déterminer leurs inoffensivités.

Un autre point à noter c'est que ces tests doivent prendre en considération les états physiologiques de l'organisme des animaux comme la présence d'un terrain d'allergie, la grossesse ainsi que le bas âge (Zahir \& Rahmani, 2020). De ce fait, il a été jugé essentiel de questionner les gens enquêtés notamment ceux trouvant que cette plante est toxique à propos des effets secondaires observés après l'emploi de $T$. articulata.

\section{Effets secondaires observés après l'emploi de $T$. articulata}

Parmi les effets secondaires observés par les enquêtés après l'emploi de $T$. articulata, figurent les diarrhées en premier lieu avec un pourcentage de $41,42 \%$ puis viennent les affections cutanées, l'avortement et l'effet de tachycardie avec des pourcentages respectifs de $25,71 \%, 11,42 \%$ et $8,57 \%$. D'autres effets ont été également

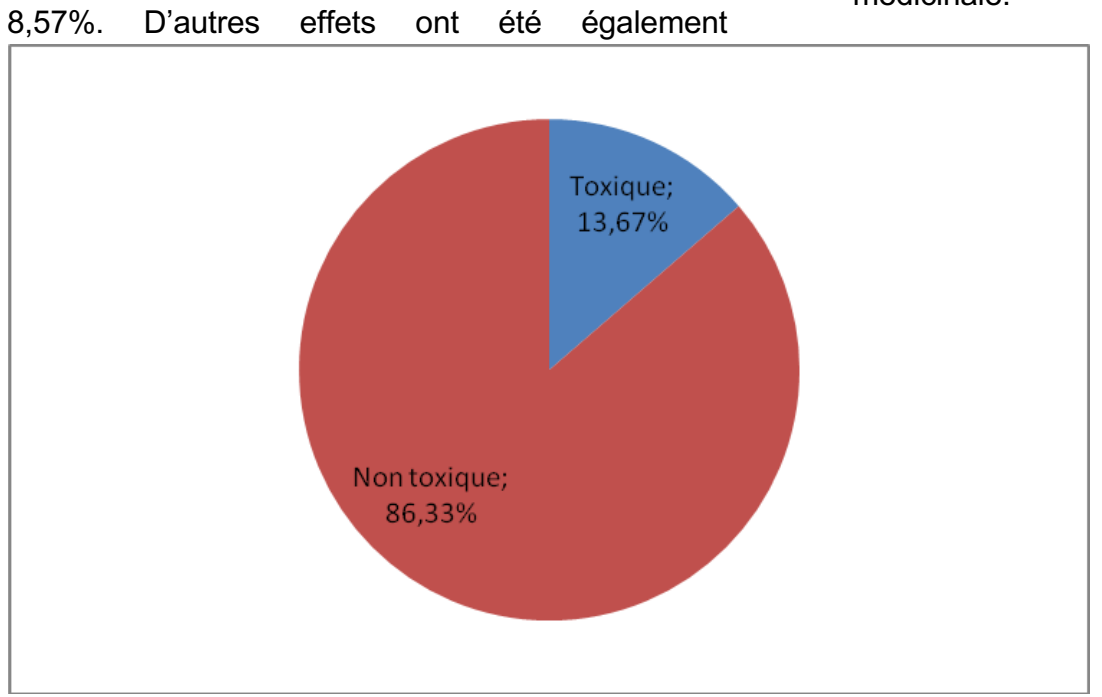

Figure 28: Evaluation de la toxicité éventuelle de $T$. articulata

Figure 28: Evaluation of the possible toxicity of $T$. articulata mentionnés représentant $10 \%$ comme l'allergie, la perte des cheveux et la sécheresse de la peau (Fig. 29).

Incontestablement, un cas clinique d'eczéma de contact très sévère a été rapporté après l'application unique d'une poudre solubilisée dans l'eau à température ambiante des feuilles et rameaux séchés de $T$. articulata. II est à rappeler que cette préparation a été utilisée en cataplasme au niveau des zones frontale, temporale et pariétale de la tête d'une jeune femme de 34 ans en raison de la fièvre due à une salmonellose mineure (Zahir \& Rahmani, 2020).

\section{Consultation d'un médecin après l'apparition des effets secondaires}

D'après la Fig. 30, on constate que plus de trois quart des enquêtés (soit $78,46 \%$ ) ne consultent pas le médecin après l'apparition des effets secondaires liés à l'emploi du thuya contre $21,55 \%$ qui le font.

\section{Conclusion}

Le présent travail se focalise principalement sur l'étude ethnobotanique de $T$. articulata dans la région de Béni Mellal-Khénifra afin de déterminer ses diverses utilisations par les habitants de cette région et de détecter les effets toxiques éventuels suite à son emploi.

Consécutivement, notre enquête a révélé une multitude de résultats montrant que $52,24 \%$ des utilisateurs de cette plante appartiennent à une tranche d'âge entre 15 et 30 ans, $57,05 \%$ sont des femmes et $51,64 \%$ ont bénéficié d'un enseignement universitaire. Ce qui rassure qu'il y aurait une bonne transmission, aux générations suivantes, de la culture d'utilisation du thuya comme plante médicinale. 


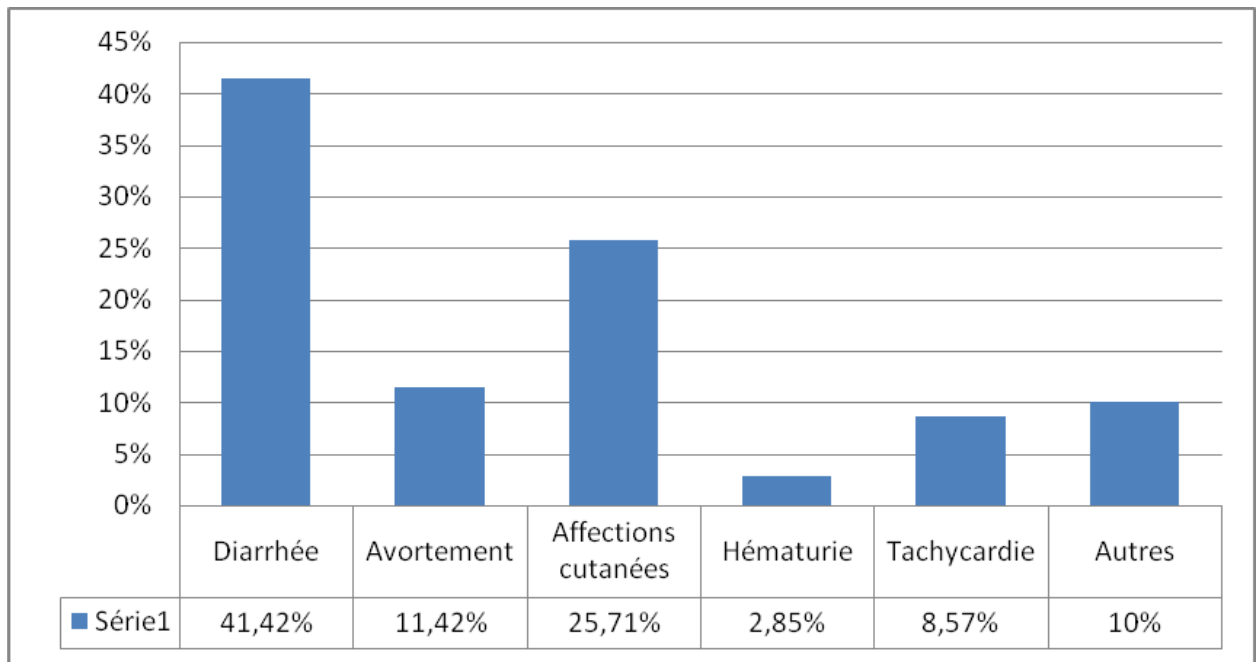

Figure 29: Effets secondaires observés après l'utilisation de T. articulata Figure 29: Side effects observed after using $T$. articulata

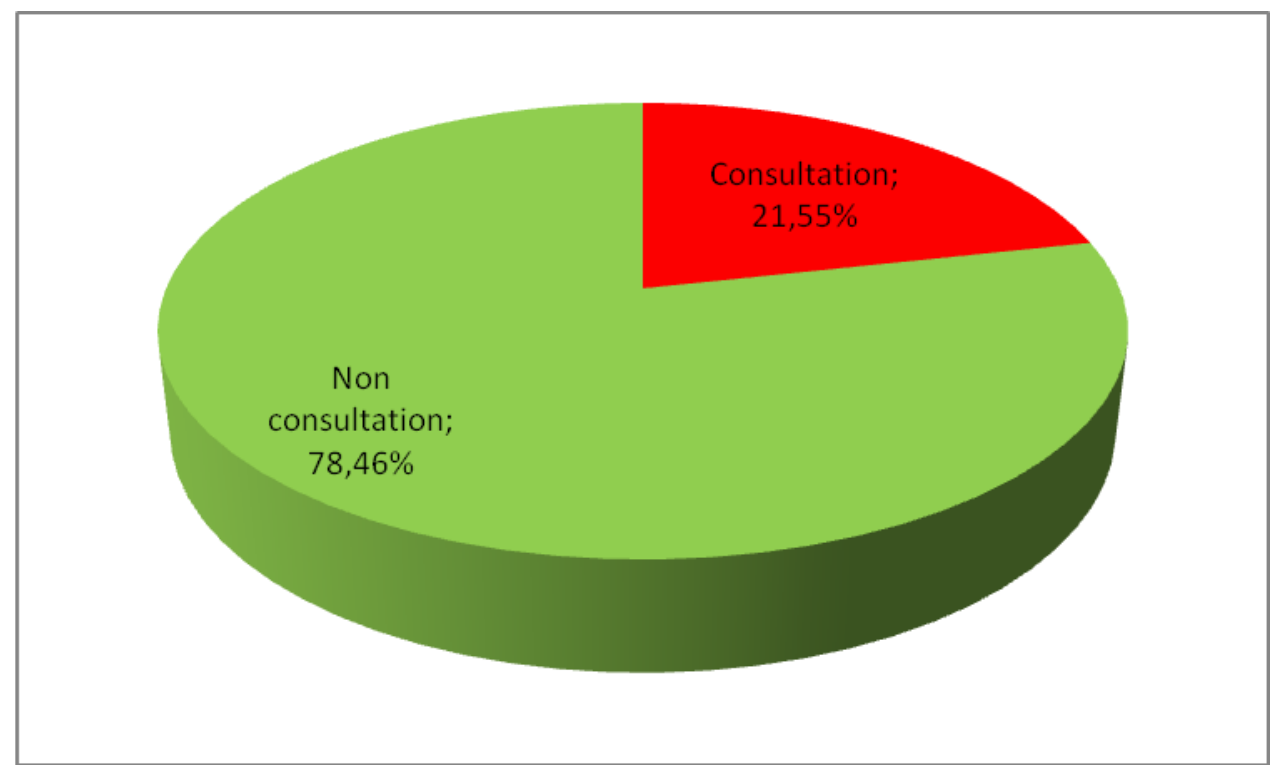

Figure 30: Consultation de médecin après l'apparition des effets secondaires

Figure 30: Doctor's consultation after the onset of side effects

Par ailleurs, le feuillage représente la partie la plus utilisée de la plante étudiée et la macération correspond au mode de préparation le plus pratiqué en thérapie, cosmétique et en alimentation.

Sur l'ensemble des maladies traitées, les affections gastro-intestinales puis la fièvre et les maux de tête représentent les pathologies les plus citées par les enquêtés.

Grâce à leurs vertus thérapeutiques, les extraits et/ ou les huiles essentielles de thuya de barbarie méritent d'être explorés amplement afin de valoriser davantage leurs activités biologiques. Mais, la plante doit aussi être soumise à des études toxicologiques et cliniques puisque quelques effets indésirables ont été mentionnés par les enquêtés suite à son emploi.
En définitif, $T$. articulata est une essence forestière largement employée par la population de la région de Béni Mellal-Khénifra. Cependant, elle doit être utilisée avec précaution et en respectant des mesures bien précises tout en tenant en compte de l'état physiologique de l'organisme (enfant, femme enceinte, présence d'un terrain d'allergie).

\section{Déclarations}

Ethiques d'approbation et consentement de participation: Tous les participants ont donné leur consentement préalable en connaissant la raison de l'étude.

Consentement à la publication: Non applicable

Conflit d'intérêt: Les auteurs déclarent qu'ils n'ont pas de conflit d'intérêt. 
Financement: Cette recherche n'a pas reçu de financement.

Contribution des auteurs: Slimane Elazaoui, Mariam Chakouri, Bouchera Naouer ont effectué des travaux sur le terrain, ainsi que l'analyse des données et le premier plan de la rédaction du manuscrit. Ilham Zahir a supervisé les travaux, traduit le résumé en anglais et amélioré la rédaction. Tous les auteurs ont lu, révisé et a approuvé la version finale de l'article avant la soumission.

\section{Références bibliographiques}

Anonyme. 2009. Quatrième Rapport National sur la Biodiversité. Royaume au Maroc Secrétariat d'Etat auprès du Ministère de l'Energie, des Mines, de l'Eau et de l'Environnement, chargé de l'Eau et de l'Environnement, ISBN:978-9954-475-39 - 3.

Azzi R, Djaziri R, Lahfa F, Sekkal FZ, Benmehdi H, Belkacem N. 2012. Ethnopharmacological survey of medicinal plants used in the traditional treatment of diabetes mellitus in the North Western and South Western Algeria. Journal of Medicinal Plants Research 6(10):2041-2050,

Bachiri L, labazi N, Daoudi A, Ibijbijen J, Nassiri L, Echchegadda G, Mokhtar F. 2015. Etude ethnobotanique de quelques lavandes marocaines spontanées. International Journal of Biological and Chemical Sciences 9(3):1308-1318.

Bammou M, Daoudi A, Slimani I, Najem M, Bouiamrine EH, Ibijbijen J, Nassiri L, 2015. Valorisation du lentisque "Pistacia lentiscus L.»: Étude ethnobotanique, Screening phytochimique et pouvoir antibactérien. Journal of Applied Biosciences 86: 7966- 7975.

Ben Akka F, Benkhnigue O, Salhi S, El Hilah F, Dahmani J, Douira A, Zidane L, 2017. Ethnobotany Study of Medicinal Plants Used in the Treatment of Respiratory Diseases in the Middle Region of Oum Rbai. International Journal of Agriculture, Environment, and Biotechnology 2(4):1460- 1468.

Bourkhiss M, Hnach L, Bourkhiss B, Stranib H. 2010. Caractérisation de l'huile essentielle de la sciure du bois de Tetraclinis articulata(vahl) Master. Bulletin de la Société Royale des Sciences de Liège 79:4-11.

Chraibi M, Fikri-Benbrahim K, Amrani M, Farah A, Bari A, Benziane Ouaritini Z, 2018. Etude éthnobotanique sur l'utilisation de Mentha pulegium, Mentha piperita et Pelargonium graveolens au nord du Maroc(Taounate) et évaluation de leur pouvoir antimicrobien. European Scientific Journal 14(24):113-133.

Direction des Eaux et Forêts, 1931. Etude sur le thuya du Maroc, les genévriers et le cyprès. Exposition Coloniale Internationale. Paris, 105 p.
Direction Générale des Collectivités Locales, 2015:La région de Béni Mellal-Khénifra, monographie générale. Rapport de ministère de l'Intérieur, 1-56.

El Azzouzi F, Zidane L. 2015. La flore médicinale traditionnelle de la région de Béni-Mellal(Maroc). Journal of Applied Biosciences 91:8493-8502.

El Jemli M, Kamal R, Marmouzi I, Doukkali Z, Bouidida EH, Touati D, Nejjari R, El Guessabi L, Cherrah Y, Alaou K. 2016. Chemical composition, acute toxicity, antioxidant and anti-inflammatory activities of Moroccan Tetraclinis articulata L. Journal of Traditional and Complementary Medicine 7(3):281-287.

El Yahyaoui O, Ait ouaaziz N, Sammama A, Kerrouri S, Bouabid B, Lrhorfi L.A, Zidane L, Bengueddour R. 2015. Etude ethnobotanique: Plantes médicinales commercialisées à la province de Laâyoune; identification et utilisation. International Journal of Innovation and Applied Studies 12(3):533-541.

Ghourri M, Zidane L, El Yacoubi H, Rochdi A, Fadli M, Douira A. 2012. Etude floristique et ethnobotanique des plantes médicinales de la ville d'El Ouatia(Maroc Saharien). Kastamonu Univ., Journal of Forestry Faculty 12(2):218-235.

Hafsé M, Fikri Benbrahim K, Saidi A, Farah A. 2013. Volatile Components and Antibacterial Profile of Essential Oils Extracted from Leaves and Twigs of Pistacia lentiscus L. British Microbiology Research Journa/ 3(4):602-611

Hajib S, Sbay H, Aafi A, Saidi S. 2013. Etat des ressources Génétiques Forestières: le royaume du Maroc. Rabat, Rapport National, $5 p$.

Fakchich J, Elachouri M. 2014. Ethnobotanical survey of medicinal plant s used by people in Oriental Morocco to manage various ailments. Journal of Ethnopharmacology.

http://dx.doi.org/10.1016/j.jep.2014.03.016i

Kadri Y, Moussaoui A, Benmebarek A. 2018. Étude ethnobotanique de quelques plantes médicinales dans une région hyper aride du Sud-ouest Algérien "Cas du Touat dans la wilaya d'Adrar». Journal of Animal \& Plant Sciences 36(2):5844-5857.

Katiri A, Barkaoui M, Msanda F, Boubaker H. 2017: Ethnobotanical Survey of Medicinal Plants Used for the Treatment of Diabetes in the Tizi n' Test Region(Taroudant Province, Morocco). Journal of Pharmacognosy and Natural Products 3(1):130.

Lahsissene $H$, Kahouadji A, Tijane M, Hseini S. 2009. Catalogue des plantes médicinales utilisées dans la région de Zaër(Maroc occidental). Lejeunia 186.

Rhattas M, Douira A, Zidane L, 2016. Étude ethnobotanique des plantes médicinales dans le Parc National de Talassemtane (Rif occidental du 
Maroc). Journal of Applied Biosciences 97 91879211.

Sadiki F, El Idrissi M, Cioanca O, Trifan A, Hancianu M, Hritcu L, Postu PA. 2018. Tetraclinis articulata essential oil mitigates cognitive deficits and brain oxidative stress in an Alzheimer's disease amyloidosis model. Phytomedicine 1-15. https://doi.org/10.1016/j.phymed.2018.10.032

Salhi N, Bouyahya A, Fettachd S, Zelloub A, Cherrah Y. 2019. Ethnopharmacological study of medicinal plants used in the treatment of skin burns in occidental Morocco(area of Rabat), South African Journal of Botany 121:128-142.

Zahir I, lyadayen O, Ettaki M, Monasef M. 2019. Place des modules dédiés à l'environnement dans le programme d'enseignement de six universités marocaines et analyse de savoir environnemental à enseigner. Journal of New Sciences, Agriculture and Biotechnology 66(2):4145-4156.

Zahir I, Rahmani A. 2020. Premier cas clinique d'eczéma de contact causé par Tetraclinis articulata. International Journal of Innovation and Applied Studies 28(2):342-346.

Zahir I, Zahir A, Taghiam I 2018. Effet antimycobactérien des plantes marocaines:revue générale. European Scientific Journal 14(36):518549. 
Tableau 1: Différentes provenances des enquêtés de la région de Béni Mellal Khénifra Table 1: Different provenances of respondents from the Beni Mellal Khénifra region

\begin{tabular}{|c|c|}
\hline Province & Commune rurale/ urbaine \\
\hline Azilal & $\begin{array}{l}\text { - Commune rurale Afourer } \\
\text { - Commune rurale Ait Abbas } \\
\text { - Commune rurale Ait M'Hamed (village Takoust) } \\
\text { - Commune rurale Ait Taguella (centre d'Ouzoud) } \\
\text { - Commune rurale Anergui } \\
\text { - Commune rurale Arbiaa Ait Attab } \\
\text { - Commune rurale Bin El Ouidane } \\
\text { - Commune rurale Bini Ayad } \\
\text { - Commune rurale Bzou } \\
\text { - Commune rurale Demnate (Iminifri, Iwaridan) } \\
\text { - Commune rurale Foum Jemaa } \\
\text { - Commune rurale Ouaouizeght } \\
\text { - Commune rurale Tabant (village Sremt) } \\
\text { - Commune rurale Tagleft } \\
\text { - Commune rurale Tanant } \\
\text { - Commune rurale Tiffert N'Ait Hamza } \\
\text { - Commune urbaine Azilal } \\
\text { - Commune urbaine Demnate }\end{array}$ \\
\hline Béni Mellal & $\begin{array}{l}\text { - Commune rurale Arhbala } \\
\text { - Commune rurale Boutferda (village Larbaa noukebli) } \\
\text { - Commune rurale Dir El Ksiba (village Imhiwach ; village Ighrem laalam) } \\
\text { - Commune rurale El Ksiba (village Gharb el alame) } \\
\text { - Commune rurale Foum El Anceur } \\
\text { - Commune rurale Foum Oudi } \\
\text { - Commune rurale Naour } \\
\text { - Commune rurale Oulad Gnaou (centre de Laayayta) } \\
\text { - Commune rurale Oulad Moussa } \\
\text { - Commune rurale Oulad Said L'Oued } \\
\text { - Commune rurale Oulad Yaich (centre Zouair) } \\
\text { - Commune rurale Oulad Youssef } \\
\text { - Commune rurale Sidi Jaber } \\
\text { - Commune rurale Taghzirt (centre Foum Zaouia) } \\
\text { - Commune rurale Tizi N’Isly } \\
\text { - Commune urbaine Béni Mellal } \\
\text { - Commune urbaine El Ksiba } \\
\text { - Commune urbaine Kasba Tadla } \\
\text { - Commune urbaine Zaouiat Cheikh }\end{array}$ \\
\hline $\begin{array}{l}\text { Fquih Ben } \\
\text { Salah }\end{array}$ & $\begin{array}{l}\text { - Commune rurale Dar Oulad Zidouh (village lamgharir) } \\
\text { - Commune rurale Hel Merbaa (centre Ouled Rguiâa) } \\
\text { - Commune rurale Oulad Zmam } \\
\text { - Commune rurale Sidi Aissa Ben Ali (village Ain Zarka, village béni Aoune, village Ouled mrah) } \\
\text { - Commune rurale Souk El Had Des Bradia (village Laajana; village Lahlalma; village El } \\
\text { Bazzaza ; village Ouled Abdellah; village Ouled Driss) } \\
\text { - Commune rurale Souk Sebt Oulad Nemma ( village Oued laabid) } \\
\text { - Commune urbaine Fquih Ben Salah } \\
\text { - Commune urbaine Oulad Ayad }\end{array}$ \\
\hline Khénifra & - Commune urbaine Khénifra \\
\hline Khouribga & $\begin{array}{l}\text { - Commune urbaine Bejaad } \\
\text { - Commune urbaine Boujniba } \\
\text { - Commune urbaine Khouribga } \\
\text { - Commune urbaine Oued Zem }\end{array}$ \\
\hline
\end{tabular}


Annexe - Questionnaire

Université Sultan Moulay Slimane

Faculté Polydisciplinaire Béni Mellal

Laboratoire Polyvalent en Recherche et Développement

\section{Licence fondamentale}

SVI

2018/2019

\section{Questionnaire}

Dans le cadre de la réalisation de notre mémoire de fin d'étude à la Faculté Polydisciplinaire de Béni Mellal, nous menons une étude sur « Etude ethnobotanique de l'utilisation de $\boldsymbol{T}$. articulata dans la région Béni Mellal Khénifra ". Nous souhaitons et nous accordons une grande importance à votre collaboration à cette recherche à travers la réponse aux questions suivantes:

A. Information personnelle à propos les enquêtés

1. Age:

Entre 1 et 15 ans $\square \quad$ Entre 15 et 30 ans $\square \quad$ Entre 30 et 100 ans

2. Sexe :

Masculin $\square \quad$ féminin

3. Niveau d'instruction :

Analphabète $\quad \square \quad$ Enseignement primaire $\quad \square \quad$ Enseignement secondaire $\square$ Enseignement universitaire $\quad \square \quad$ Enseignement non-formel

4. Lieu de résidence :

Ville $\quad \square \quad$ Village $\square \quad$ Montagne

B. Usage de T. articulata (arâar)

1. Avez-vous entendu à propos la plante arâar ?

Oui $\square$ Non

2. L'avez-vous déjà utilisé ?

Oui $\square$ Non

3. Quels sont les domaines d'utilisation? 3.1 Thérapie

Affections gastro intestinales $\square \quad$ Maux de tête $\square$

Fièvre

Cicatrisation $\square$ Affections articulaires

Problèmes de stomatologies et d'ondotologie $\square$ Affections cutanées $\square$

oro-pharyngées $\square \quad$ Maladies métaboliques (diabète, la goutte, le cholestérolémie,.....

Affections respiratoires $\square$ Douleurs rénales $\square \quad$ Affections cardio vasculaires $\square \quad$ Affections uro génitales

$\square$ Détoxification Autres $\square$ précisez

3.2 Cosmétique

Shampoing $\square$ Goudron végétal 3.3 Alimentation

Aromatisant de petit lait 3.4 Décoration

OEuvres d'art $\underline{3.5 \text { Industrie }}$

Papeterie

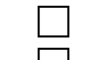

Menuiserie

Tannerie

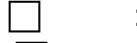

Lotion de tout le corps (rhasoul)

Autres

3.6 Pâturage

$\square \quad$ Autres

Face de jardin $\quad \square \quad$ Autres

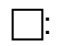

$\square \quad$ Industrie de porcelaine

Autres
Exportation de bois

précisez.

4. Quelle est la partie utilisée de la plante:

4.1 En thérapie

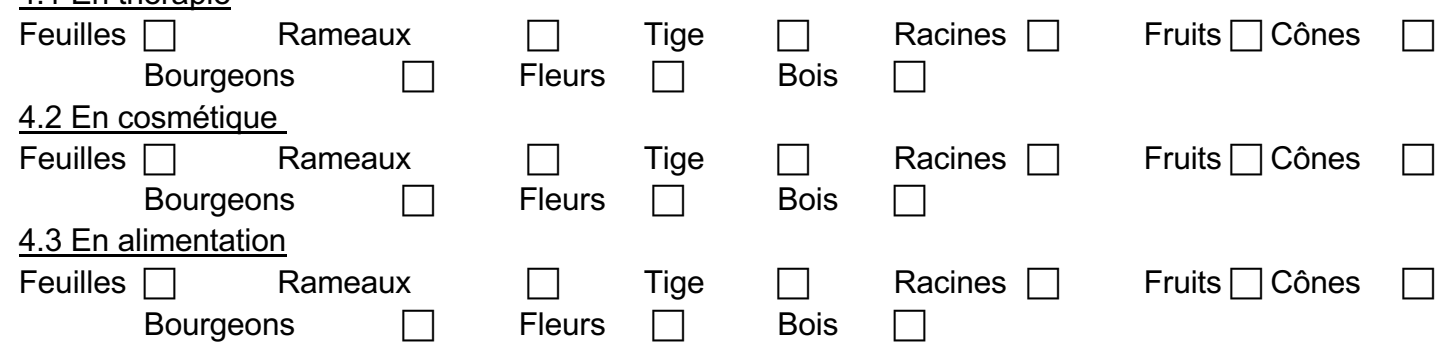

5. Est-ce que la plante est utilisée sous forme :

Fraiche $\square \quad$ Séchée 
6. Quelle est la forme d'emploi la plus utilisée ?

Poudre $\quad \square \quad$ Huile essentielle $\square \quad$ Extrait $\square \quad$ Tisane $\square$ Autres $\square$ précisez.............

7. Quel est le mode de préparation à base de T. articulata? Pourquoi

7.1 En thérapie :
Macération
Infusion
Décocté
Cataplasme
7.2 En cosmétique: Macération
7.3 En alimentation: Macération $\square \quad$ Infusion $\square \quad$ Décocté $\square$

8. Quelle est la voie d'administration de la plante en thérapie:
Orale
Cutanée
Autres

9. Quelle est la dose utilisée ?

Pincée $\quad \square \quad$ Poignée $\square \quad$ Cuillère $\square$ Dose précise

10. Quelle est le nombre de prise par jour?
1 fois/j
2 fois/j $\square \quad 3$ fois/j
Autres $\square$ précisez.

11. Quelle est la période de la thérapie ?

12. Comment évaluez-vous le résultat obtenu après l'emploi de $T$. articulata en thérapie ?

Résultat satisfaisant $\quad \square$ Sans résultat $\quad \square \quad$ Résultat néfaste (aggravation de cas apparition d'effet secondaire

13. Comment évaluez-vous le résultat obtenu après l'emploi de $T$. articulata en cosmétique ? $\begin{array}{lll}\text { 14. Résultat satisfaisant } & \square \text { Sans résultat } & \square\end{array}$

C. Toxicité

1. Est-ce que cette plante est toxique ? Oui $\square \quad$ Non

2. Quels sont les effets secondaires observés? Diarrhée Avortement

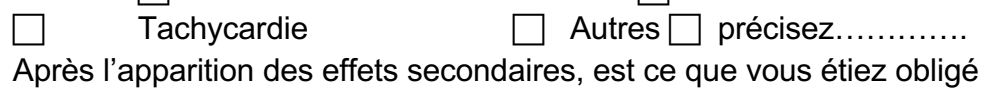
Autres $\square$ précisez...

Affections cutanées Hématurie

3. Après l'apparition des effets secondaires, est ce que vous étiez obligé de consulter un médecin ? Oui

4. Quels conseils vous pouvez donner aux utilisateurs de T. articulata? 